\title{
Quantum confinement induced metal-insulator transition in strongly correlated quantum wells of $\mathrm{SrVO}_{3}$ superlattices
}

\author{
A. D. N. James $\odot,{ }^{1}$ M. Aichhorn, ${ }^{2}$ and J. Laverock $\odot^{1, *}$ \\ ${ }^{1}$ H. H. Wills Physics Laboratory, University of Bristol, Tyndall Avenue, Bristol BS8 1TL, United Kingdom \\ ${ }^{2}$ Institute of Theoretical and Computational Physics, TU Graz, NAWI Graz, Petersgasse 16, 8010 Graz, Austria
}

(Received 28 May 2020; revised 7 May 2021; accepted 10 May 2021; published 26 May 2021)

\begin{abstract}
Dynamical mean-field theory (DMFT) has been employed in conjunction with density functional theory $(\mathrm{DFT}+\mathrm{DMFT})$ to investigate the metal-insulator transition (MIT) of strongly correlated $3 d$ electrons due to quantum confinement. We shed light on the microscopic mechanism of the MIT and previously reported anomalous subband mass enhancement, both of which arise as a direct consequence of the quantization of $\mathrm{V}$ $x z(y z)$ states in the $\mathrm{SrVO}_{3}$ layers. We therefore show that quantum confinement can sensitively tune the strength of electron correlations, leading the way to applying such approaches in other correlated materials.
\end{abstract}

DOI: 10.1103/PhysRevResearch.3.023149

\section{INTRODUCTION}

In strongly correlated quantum materials, the interaction energy between electrons is comparable with their kinetic energy, leading to many-body behavior and the emergence of qualitatively new phenomena [1]. For example, transition metal oxides host properties as diverse as colossal magnetoresistance, high-temperature superconductivity, and Mott insulating phases, each of which have huge potential for future device and technology applications [2,3]. More recently, substantial advances in the quality and control of layer-by-layer growth methods have facilitated designed transition metal oxide heterostructures and superlattices (SLs), often focusing on emergence at interfaces and/or surfaces [4]. Here, we show that correlated electronic behavior may be delicately tuned by quantum confinement, which narrows the effective bandwidth of the correlated quantum well (QW) subbands and establishes another means to tune physical properties to suit applications. We illustrate this by driving the prototypical correlated metal $\mathrm{SrVO}_{3}$ through a metal-insulator transition (MIT) to a Mott insulating phase, in excellent quantitative agreement with the experimental spectral function extracted from recent spectroscopic measurements [5].

In the bulk, $\mathrm{SrVO}_{3}$ is a well-characterized correlated metal [6]. Sharp 3d quasiparticle (QP) bands at low excitation energies lead to a well-defined Fermi surface [7], while localized states form incoherent Hubbard sidebands at an energy scale comparable with the Coulomb repulsion parameter $U$ $[8,9]$. Together, these yield the familiar three-peaked spectral function $[3,10]$. While density functional theory (DFT) often adequately describes QP states (once renormalization is ac-

\footnotetext{
*j.laverock@bristol.ac.uk

Published by the American Physical Society under the terms of the Creative Commons Attribution 4.0 International license. Further distribution of this work must maintain attribution to the author(s) and the published article's title, journal citation, and DOI.
}

counted for), it is not capable of capturing the many-body behavior, e.g., Hubbard sidebands are completely absent. Dynamical mean-field theory (DMFT), on the other hand, can describe all of the onsite local correlations $[3,10]$ and has been well-tested on $\mathrm{SrVO}_{3}$ with very good results, including the energetics and spectral weight of Hubbard sidebands and QP renormalization $[3,8,11,12]$. Meanwhile, a recent $G W+$ DMFT study (where $G W$ refers to Hedin's $G W$ approximation [13]) has suggested that bulk $\mathrm{SrVO}_{3}$ is weakly correlated [14].

The importance of electron correlations in a system may be gauged in terms of the ratio $U / W$, where $W$ is the bandwidth. This ratio is known to be significantly enhanced at the $\mathrm{SrVO}_{3}$ surface $[15,16]$ and in few-layer systems $[17,18]$. However, such systems have reduced coordination at the surface, which is often complicated by reconstruction and relaxation [16]. For example, an insulating phase has been observed in $\mathrm{SrVO}_{3}$ thin films for thicknesses $<\sim 6$ unit cells, which was attributed to dimensional crossover due to reduced coordination [17,19]. In bilayer $\mathrm{SrVO}_{3}$, a DFT + DMFT study has established that a Mott insulator emerges due to crystal field (CF) effects [18]; similar CF-induced insulating phases are predicted in other vanadates [20-23]. Although QW structures are well known in semiconducting [24] and free electronlike [25] materials, their application to correlated $3 d$ metals has only recently been realized in a select few transition metal oxides [26,27], including in few-layer $\mathrm{SrVO}_{3}$ thin films [28]. However, the precise nature of these states, including their unusual subband renormalization $[28,29]$, is not yet well understood, although this is essential to exploit their properties.

Here, we show that quantum confinement can be employed to precisely control strongly correlated electron behavior. We reveal the microscopic mechanism involved using a combination of DFT, DFT + DMFT, and tight-binding (TB) models applied to the prototypical correlated oxide $\mathrm{SrVO}_{3}$, which we embed within layers of $\mathrm{SrTiO}_{3}$ in a SL for direct comparison with previous experimental results [5]. Our results accurately reproduce the trends of the experimental data and reveal that the microscopic mechanism for the MIT in $\mathrm{SrVO}_{3} / \mathrm{SrTiO}_{3}$ SLs is due to quantum confinement. Our results also shed light 

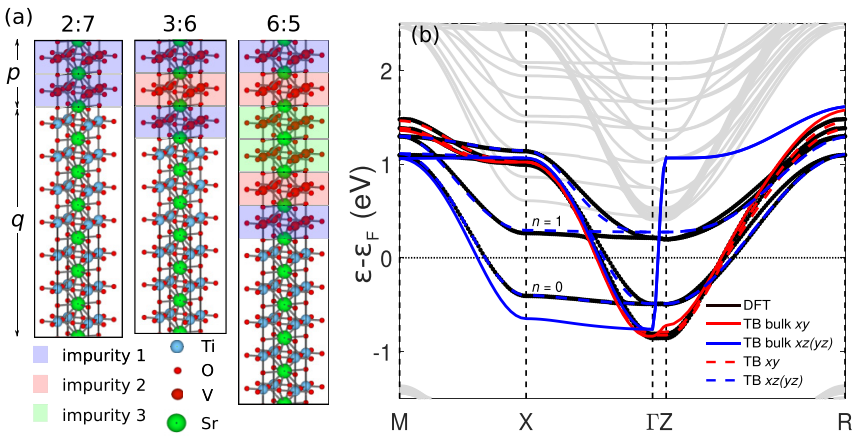

FIG. 1. (a) Schematic diagram of the superlattice (SL) structures, where $p$ and $q$ represent the number of $\mathrm{SrVO}_{3}$ and $\mathrm{SrTiO}_{3}$ layers, respectively. The SL unit cells used in the density functional theory (DFT) are indicated by the black boxes. The color-coded layers illustrate the impurities used in the dynamical mean-field theory (DMFT) calculation. (b) Quantized tight-binding (QTB) bands for the 2:7 SL. The colored dashed lines indicate the intrinsic bulk bands from which the quantized bands are derived (see text). The discrete energies from the DFT calculation are shown for comparison (both $\mathrm{V}$ and Ti $t_{2 g}$ bands are given). The gray bands are non- $\mathrm{V} t_{2 g}$.

on previous observations of anomalous mass enhancement in $\mathrm{SrVO}_{3}$ QWs [28,29], which can be naturally explained as consequences of quantization. These results present a means of controlling correlated electronic properties using quantum confinement as a control parameter. We demonstrate that the flexibility and range of control is much greater than that achievable through conventional means of adjusting the bandwidth, e.g., substitutional doping of single crystals. The possibility of controlling the bandwidth of a correlated material, and therefore the importance of electron correlations, opens up opportunities toward manipulating the electronic structure of this rich class of materials [30].

\section{THEORETICAL METHODS}

DFT calculations were performed using the all-electron full potential augmented plane-wave ELK package [31] within the local density approximation (LDA). Details about the DFT calculations are provided in Appendix B. The results are in excellent agreement with previous pseudopotential calculations [5]. The structures of the $\left(\mathrm{SrVO}_{3}\right)_{p} /\left(\mathrm{SrTiO}_{3}\right)_{q} \mathrm{SLs}$ are shown in Fig. 1(a) for the three SLs investigated here, with $p: q=2: 7,3: 6$, and 6:5. These were chosen for direct comparison with previous experimental results [5,32]. Experimental lattice parameters were used: the in-plane parameters were those of the $\left(\mathrm{LaAlO}_{3}\right)_{0.3}\left(\mathrm{Sr}_{2} \mathrm{TaAlO}_{6}\right)_{0.7}$ (LSAT) substrate $a=b=3.868 \AA$, and out-of-plane parameters were $c_{2: 7}=4.00 \AA, c_{3: 6}=3.97 \AA$, and $c_{6: 5}=3.92 \AA$. Additional calculations were performed using relaxed atomic positions and lattice parameters, leading to similar qualitative behavior. Even in the absence of the SL heterostructure, the different in- and out-of-plane lattice parameters weakly break the $\mathrm{V} t_{2 g}$ degeneracy into $3 d x y$ and $x z(y z)$ orbitals.

To interpret our results, the LDA results for each SL were parameterized using a quantized Bohr-Sommerfeld TB (QTB) model [25] (see Appendix C for further details). In this model, the shape of the TB bands was fixed to that of bulk $\mathrm{SrVO}_{3}$, and the free parameters represent the band centers, bandwidths, and quantization parameters. In this way, CF splitting and band narrowing between $x y$ and $x z(y z)$ orbitals can be fully captured. The results of fitting this model to the full ELK calculation is shown in Fig. 1(b) for the 2:7 SL, demonstrating the excellent agreement between the two. The quantized nature of the $\mathrm{V} x z(y z)$ orbitals is clearly evident, leading to two subbands $(n=0,1)$ originating from the two $\mathrm{SrVO}_{3}$ layers. In Fig. 1(b), we also show the "intrinsic" TB bands, which correspond to bulklike bands before the quantization conditions are applied and represent the intrinsic three-dimensional (3D) electronic structure from which the QW states emerge.

For the DMFT calculation, we employed the TRIQS/ CTHYB continuous-time quantum Monte Carlo (CTQMC) solver [33] in the TRIQS library [34], along with the previously used Hubbard-Kanamori interaction Hamiltonian $\beta=$ $40 \mathrm{eV}^{-1}(\sim 290 \mathrm{~K}), J=0.75 \mathrm{eV}$, and fully localized limit double counting term $[18,20,21]$. The DMFT cycle requires multiple impurities depending on the SL structure, as illustrated schematically in Fig. 1(a). The systems investigated correspond to a single impurity for the 2:7 SL and bulk calculations, and two and three impurities for the 3:6 and 6:5 SLs, respectively. In each case, the impurities are considered independent of one another. Only the (projected) $\mathrm{V} t_{2 g}$ states (using Wannier projectors [35]) were treated as correlated in the DMFT calculation.

As in recent studies [36], the results presented use the fully charge self-consistent DFT + DMFT technique as implemented in the TRIQS/DFTTools library [37]. We obtain similar results with the one-shot approach with the exception of the orbital charges (see Appendix D), which is consistent with other studies [20-22]. For each SL structure, $U$ was varied in the range $5.5-6.25 \mathrm{eV}$, and $U_{\mathrm{MIT}}$ was located, corresponding to the $U$ at which the SL becomes insulating. In Fig. 2(a), we show the orbitally averaged QP residue $\bar{Z}=\left(\Sigma_{i} Z_{i}\right) / N$ for each SL, showing how $U_{\text {MIT }}$ increases by $\sim 0.2 \mathrm{eV}$ for each SL. Here, $Z_{i}$ was determined from the $i$ th orbital self-energy on the Matsubara frequency axis.

\section{RESULTS}

We begin by ensuring that our DFT + DMFT calculations accurately describe the experimental system. In Figs. 2(b)2(d), we compare quantities extracted from x-ray absorption spectroscopy (XAS) and resonant inelastic x-ray scattering (RIXS) experiments [5] with the corresponding theoretical quantities from our DFT + DMFT calculations for $U=$ $5.7 \mathrm{eV}$, chosen to reproduce the phenomenological behavior of the experiments. As demonstrated by Figs. 2(b)-2(d), our DFT + DMFT calculations not only capture the qualitative behavior, but also yield excellent quantitative agreement with the experiment trends. Our chosen $U$ value is in good agreement with previous constrained random phase approximation calculations of relaxed freestanding layers of $\mathrm{SrVO}_{3}$ [18]. Additional calculations using the $G W$ method demonstrate the insulating state evolves due to strong electron correlation and not due to the effects of nonlocal exchange (see Appendix D). We note that, although the 2:7 SL is macroscopically insulating, it has a small QP spectral weight in the spectroscopic experiments due to properties of the sample. 

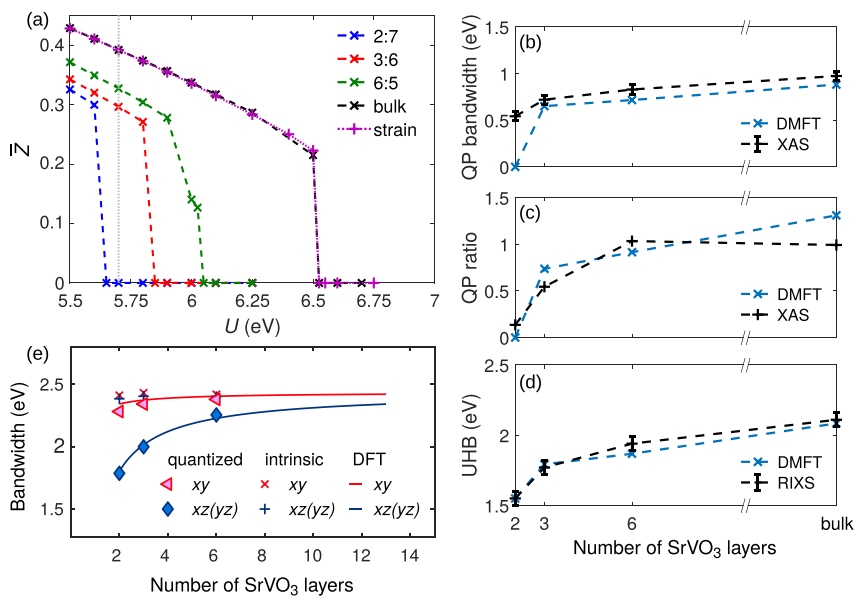

FIG. 2. (a) The orbitally averaged quasiparticle (QP) residue $\bar{Z}$ for each of the $p: q$ superlattices (SLs). Also shown are the results of bulk and $1 \%$ strained calculations (see text). The gray dotted line indicates the value of $U=5.7 \mathrm{eV}$ used for subsequent calculations. (b)-(d) Comparison with experimental quantities from x-ray absorption spectroscopy (XAS) and resonant inelastic x-ray scattering (RIXS) data (see Appendix A) [5]: (b) QP bandwidth; (c) spectral weight within QP states compared with upper Hubbard band (UHB) states; (d) energy of UHB [the dynamical mean-field theory (DMFT) results have been rigidly shifted to match at the $2: 7 \mathrm{SL}$. (e) The bandwidths of the SLs from the density functional theory (DFT) calculation and quantized tight-binding (QTB) model. Also shown are the intrinsic (bulk) bandwidths of the QTB model.

Insight into the microscopic mechanism for the MIT can be obtained by analyzing the QTB model for each SL. In Fig. 2(e), the bandwidths of the SLs from the DFT calculations are shown alongside those from the QTB model. While the in-plane $x y$ orbitals experience a slight narrowing for thinner $\mathrm{SrVO}_{3} \mathrm{SLs}$, the overall band narrowing of the (quantized) out-of-plane $x z(y z)$ orbitals is substantial, leading to a bandwidth reduction of $\sim 70 \%$ for the $2: 7 \mathrm{SL}$ [5]. As expected from the quality of the fits in Fig. 1(b), good agreement is observed between the QTB and DFT results. However, this behavior is not captured at all by the intrinsic bandwidths, which correspond to the effective 3D bands of the QTB model before quantization. These results demonstrate that the band narrowing in Fig. 2(e) is due to quantization of the $x z(y z)$ orbitals, which has a more pronounced impact for thinner $\mathrm{SrVO}_{3}$ layers. The band narrowing of the thinner SLs leads to a greater $U / W$ ratio, which results in stabilization of the insulating phase, as illustrated by the DFT + DMFT calculations shown in Fig. 2(a).

A previous DMFT study has attributed CF effects as being the principle factor driving the MIT in bilayer $\mathrm{SrVO}_{3}$ on $\mathrm{SrTiO}_{3}$ [18], and it is pertinent to ask what role, if any, the CF plays in our system. In Ref. [18], strain induced by the $\mathrm{SrTiO}_{3}$ substrate led to a lowering of the $x y$ orbitals by $180 \mathrm{meV}$ due to the CF. In contrast, we find a CF splitting of $\leqslant 51 \mathrm{meV}$ in favor of the $\mathrm{V} x z(y z)$ bands in our SLs, in part owing to the lower strain imparted by the LSAT substrate. To reproduce the effects of this $\mathrm{CF}$, we have calculated strained $\mathrm{SrVO}_{3}$ with a volume-conserving strain of $1 \%$, which leads to similar CF splitting of $53 \mathrm{meV}$ (see Appendix D for more details).
This 3D system, which reproduces the CF levels of our SLs but without the quantization effects, is shown in Fig. 2(a) and shows very similar behavior to the bulk cubic system. Therefore, we confidently rule out CF effects as a dominant factor in our SLs.

Finally, we discuss the correlated behavior of the quantized electron states, taking the 6:5 SL as an example. Figures 3(a)3(c) show the orbital- and layer-resolved occupation number $n_{e}$, QP residue $Z$, and spectral weight at the Fermi level $A(\omega=0)$ as a function of $U$, illustrating the transition to the insulating phase at $U=6.05 \mathrm{eV}$ for this SL. As presented in Fig. 3(a), each layer (impurity) exhibits a sizable orbital polarization in favor of the $x y$ orbitals, which is exaggerated both at the outer (interface) layer and in the insulating phase. This behavior is consistent across all SLs and originates from a small polarization in the DFT calculations due to the local CF, which is subsequently amplified in the DMFT cycle (see Appendix D). The outer layer (impurity 1) is significantly "more correlated," experiencing a smaller QP residue $Z$ than the other layers [Fig. 3(b)], corresponding to a greater renormalization factor $1 / Z$. Near the MIT, this leads to a collapse in the spectral weight at $\omega=0$ of the interface layer [Fig. 3(c)]. In this sense, the more correlated interface layer simultaneously triggers the MIT in the remaining layers, in much the same way as suggested for $\mathrm{SrVO}_{3}$ bilayers in Ref. [18].

The DMFT spectral functions $A(\omega)$ and $A(\mathbf{k}, \omega)$ are shown in Figs. 3(d)-3(f) and Figs. 3(h)-3(j) for $U=5.7,6.025$, and $6.05 \mathrm{eV}$, respectively, and were calculated using the maximum entropy method within TRIQS [38]. The renormalization of the $\mathrm{V}$ bands increases from a factor of $1 / \bar{Z} \approx 3.5$ at $U=$ $5.7 \mathrm{eV}$ to $\approx 8$ just below the MIT $\left(U_{\mathrm{MIT}}=6.05 \mathrm{eV}\right)$ before the QP spectral weight vanishes in the insulating phase [Fig. 3(f)]. The renormalization of the $\mathrm{V}$ bands is accompanied by a lowering in their energy with respect to $\mathrm{O}$ and Ti bands, leading to pronounced energy separation of $\mathrm{O} / \mathrm{Ti}$ bands and metallic $\mathrm{V} t_{2 g}$ QPs. Nevertheless, the quantized subband structure remains clear in the metallic solutions, leading to genuinely strongly correlated QWs. At $U=5.7 \mathrm{eV}$, the lowest three $y z$ subbands are occupied at $X$ and $\Gamma$, like the DFT; however, by $U=6.025 \mathrm{eV}$, only the lowest subband is occupied, indicating the quantized system undergoes a correlation-induced Lifshitz transition before the MIT. Although the correlated orbitals remain relatively sharp throughout near $\omega=0$, implying long-lived QPs with a well-defined Fermi surface, the lifetime rapidly broadens away from $\varepsilon_{\mathrm{F}}$, in line with a strongly correlated Fermi liquid (see inset to Fig. 4). We also note that the incoherent upper Hubband band (UHB), visible $>1 \mathrm{eV}$ in [Fig. 3(j)], exhibits rather strong momentum dependence, visible in previous bulk DMFT calculations [11,39]. Unexpectedly, certain (uncorrelated) Ti states also show pronounced broadening, e.g., the $n=1 \mathrm{Ti} y z$ orbital indicated in Fig. 3(h), which is almost completely smeared out at $U=6.025 \mathrm{eV}$ [Fig. 3(i)]. This arises due to the spatial penetration of the $\mathrm{Ti}$ wave functions at the interface into the correlated $\mathrm{SrVO}_{3}$ layers, leading to broadening of those subbands that have significant weight at the interface (see Appendix B). This surprising sensitivity of the Ti states may help to explain recent RIXS results of the $\mathrm{SrTiO}_{3}$ layers of $\mathrm{SrVO}_{3} / \mathrm{SrTiO}_{3} \mathrm{SLs}$, where a sudden change in the delocalized 

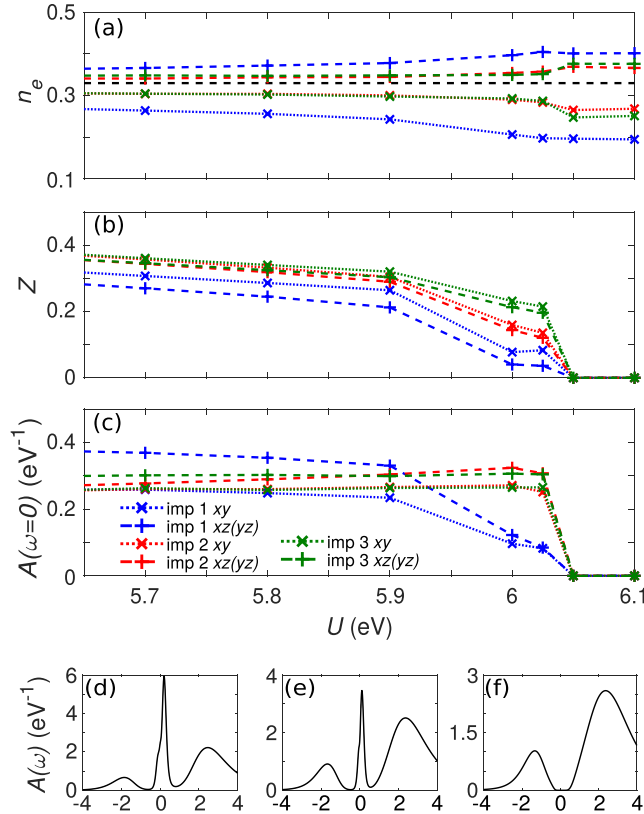

$\omega(\mathrm{eV})$

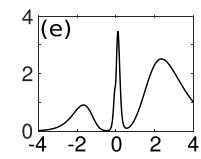

$\omega(\mathrm{eV})$

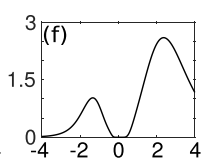

$\omega(\mathrm{eV})$
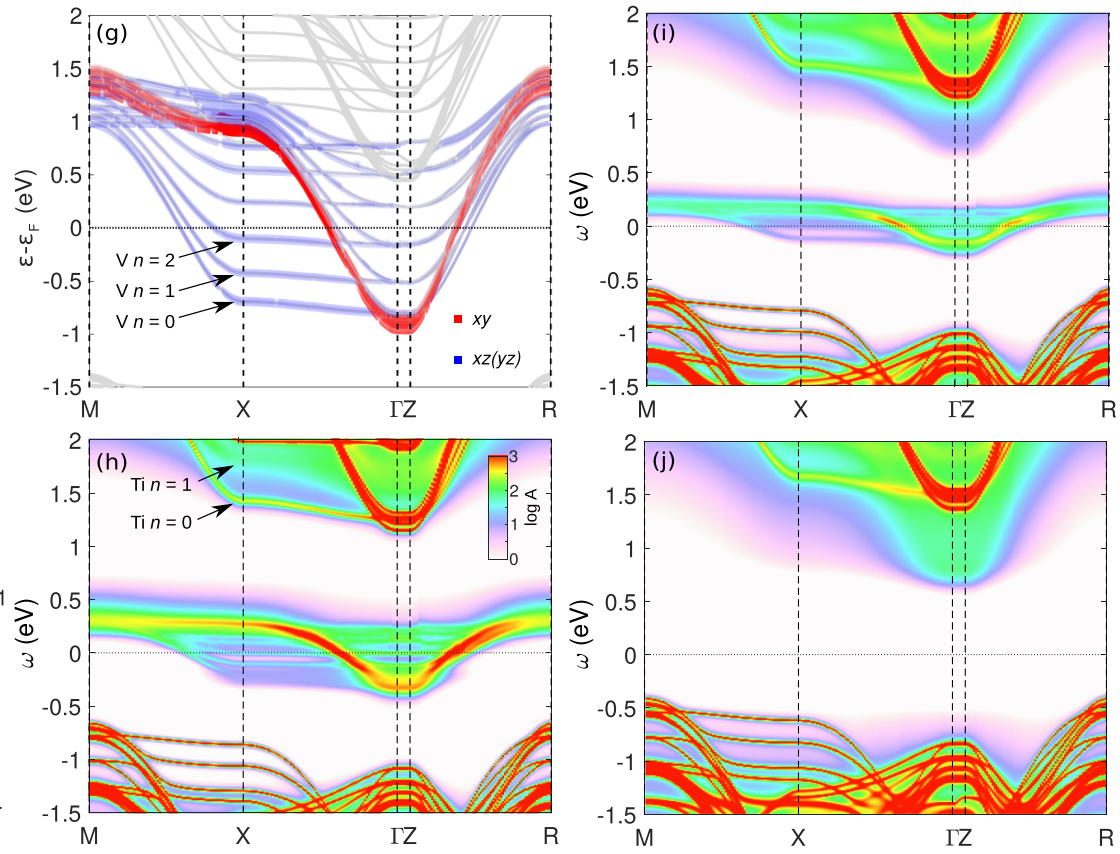

FIG. 3. Correlated quantum well (QW) electronic structure. (a) Occupation number $n_{e}$, (b) quasiparticle (QP) residue $Z$, and (c) spectral function at the Fermi level $[A(\omega=0)]$, determined directly from the imaginary time Green's function, of all impurity (imp) correlated orbitals from density functional theory (DFT) + dynamical mean-field theory (DMFT) across the metal-insulator transition (MIT) for the 6:5 superlattice (SL). (d)-(f) The momentum-integrated $\mathrm{V} t_{2 g}$ spectral function $A(\omega)$ for $U=5.7,6.025$, and $6.05 \mathrm{eV}$, respectively. (g) DFT band structure of the 6:5 SL, with $\mathrm{V} t_{2 g}$ band characters shown. (h) DFT + DMFT momentum-resolved spectral function $A(\mathbf{k}, \omega)$ of the 6:5 SL for $U=5.7 \mathrm{eV}$, showing directly the renormalization of the correlated bands. (i) and (j) DFT + DMFT spectral function close to the MIT for $U=6.025$ and $6.05 \mathrm{eV}$, respectively.

Ti $3 d$ carrier density was observed coincident with the MIT of the $\mathrm{SrVO}_{3}$ layers [40].

We finish by discussing some important implications of our results. Anomalous mass enhancement has been reported in $\mathrm{SrVO}_{3}$ QWs, whereby shallower occupied subbands were found to have a larger mass enhancement $\left(m^{\star}=m / Z_{v}\right)$ than subbands at deeper energies [28]. Subsequent theoretical [41] and experimental [29] studies argued the anomalous enhancement was a consequence of a combination of electron

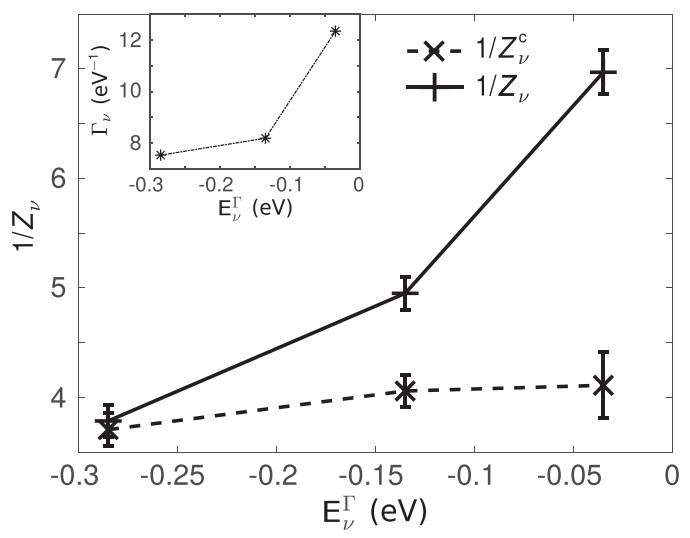

FIG. 4. The total mass enhancement factor of each subband $1 / Z_{v}$ of the 6:5 SL at $U=5.7 \mathrm{eV}$ shown alongside the correlation-only mass enhancement factor $1 / Z_{v}^{\mathrm{c}}$, evaluated along $X-M$. The abscissa represents the bottom energy of the subband. Error bars indicate the estimated error in determining these quantities. The inset shows the subband lifetime $\Gamma_{v}$. All lines are guides to the eye. correlation effects and the reduced dimensionality of the surface. To address this, we compare the total mass enhancement of a particular quantized subband $1 / Z_{v}$ with that due solely to electron correlation $1 / Z_{v}^{\mathrm{c}}$, both of which are obtained through analysis of the Fermi velocities (see Appendix D for more details). The trend in the total mass enhancement factor $1 / Z_{\nu}$, which includes both correlation effects and band effects due to quantization, qualitatively reproduces the experimental data [28] rather well, but this behavior is only very weakly present in $1 / Z_{v}^{c}$. Therefore, in our SLs, the origin of the mass enhancement is firmly due to $k_{z}$ sampling introduced by quantization, as has been observed in other systems [27]. While reduced coordination at the surface [16,41], surface reconstruction [42], or specific $k_{z}$ sampling due to an asymmetric phase shift at the vacuum $[27,43]$ may play additional roles in specific surface-terminated systems, our results reveal quantization as the primary source of anomalous mass enhancement in embedded (or capped) few-layer $\mathrm{SrVO}_{3}$.

\section{CONCLUSIONS}

In summary, our results establish quantum confinement engineering as a sensitive method to tune the correlated electron behavior of $3 d$ electron systems. We demonstrate our approach using SLs of few-layer $\mathrm{SrVO}_{3}$ embedded in $\mathrm{SrTiO}_{3}$ : this system exhibits an MIT due to a reduction in bandwidth by varying the number of $\mathrm{SrVO}_{3}$ layers. The microscopic mechanism we reveal is a direct consequence of quantum confinement and is distinct from previous studies invoking CF effects [18] or dimensionality [17], demonstrating the 
excellent versatility of the MIT in $\mathrm{SrVO}_{3}$ with respect to different control parameters. Like Ref. [18], we find that the outer layers at the interface of the QW are more strongly correlated and trigger the transition in the rest of the layer. Our interpretation can also naturally explain the anomalous mass enhancement previously reported in angle-resolved photoemission spectroscopy measurements $[28,29]$. Together, these results demonstrate the potency of employing quantum confinement as a tuning parameter for correlated electron behavior in engineered SLs. This approach also has the significant benefits that surface effects are avoided and that large (bulklike) volumes of the system may respond to external stimuli. Aside from the expected improved performance of such a $\mathrm{SrVO}_{3}$ device as a Mott transistor [18], quantized SLs made of other correlated materials are likely to show improved properties, e.g., superconducting devices in cuprate SLs [44], conductivity in nickelate SLs [45], or spintronic devices [46].

Data are available at the University of Bristol data repository, data.bris, at [47].

\section{ACKNOWLEDGMENTS}

A.D.N.J. acknowledges funding and support from the Engineering and Physical Sciences Research Council (EPSRC) Centre for Doctoral Training in Condensed Matter Physics (CDT-CMP), Grant No. EP/L015544/1. M.A. acknowledges financial support from the Austrian Science Fund FWF, Grant No. Y746. Calculations were performed using the computational facilities of the Advanced Computing Research Centre, University of Bristol [48]. The authors would like to thank Dr. C. Bell, Dr. G. Kraberger, and Prof. S. B. Dugdale for their useful discussions and contributions related to this paper. The VESTA package [49] has been used in the preparation of some figures.

\section{APPENDIX A: COMPARISON WITH EXPERIMENTAL QUANTITIES}

Figure 5 illustrates the main experimental spectroscopic results on the SLs [5,32], in which transport measurements established that the 2:7 and 3:6 SLs were insulating, whereas the 13:4 SL was metallic. The 6:5 SL was found to be metallic at room temperature, with an MIT at low temperature. The experimental results (performed at room temperature) show the evolution in correlated electron behavior extracted from XAS and RIXS.

For completeness, we briefly outline the experimental properties and how they were extracted here. The metallicity [Fig. 5(a)] was extracted from both XAS and RIXS as the leading edge of the $\mathrm{O} K$ edge XAS and from the intensity of the quasi-elastic peak in V $L$ edge RIXS. The QP bandwidth [Fig. 5(b)] was extracted from the $\mathrm{SrVO}_{3}$ layer contribution to the $\mathrm{O} K$ edge XAS as the full width at half-maximum of the QP peak. The QP spectral weight [Fig. 5(c)] was also extracted from the $\mathrm{SrVO}_{3}$ layer contribution to the $\mathrm{O} K$ edge $\mathrm{XAS}$ as the ratio of the area under the QP peak to the total area under unoccupied V $3 d$ states (i.e., the sum of QP, UHB, and $e_{g}$ spectral weights). Occupied states are not accessible in $\mathrm{O} K$ edge XAS. Finally, the UHB energy [Fig. 5(d)] is accessible to both XAS and RIXS. From O $K$ edge XAS, the UHB peak

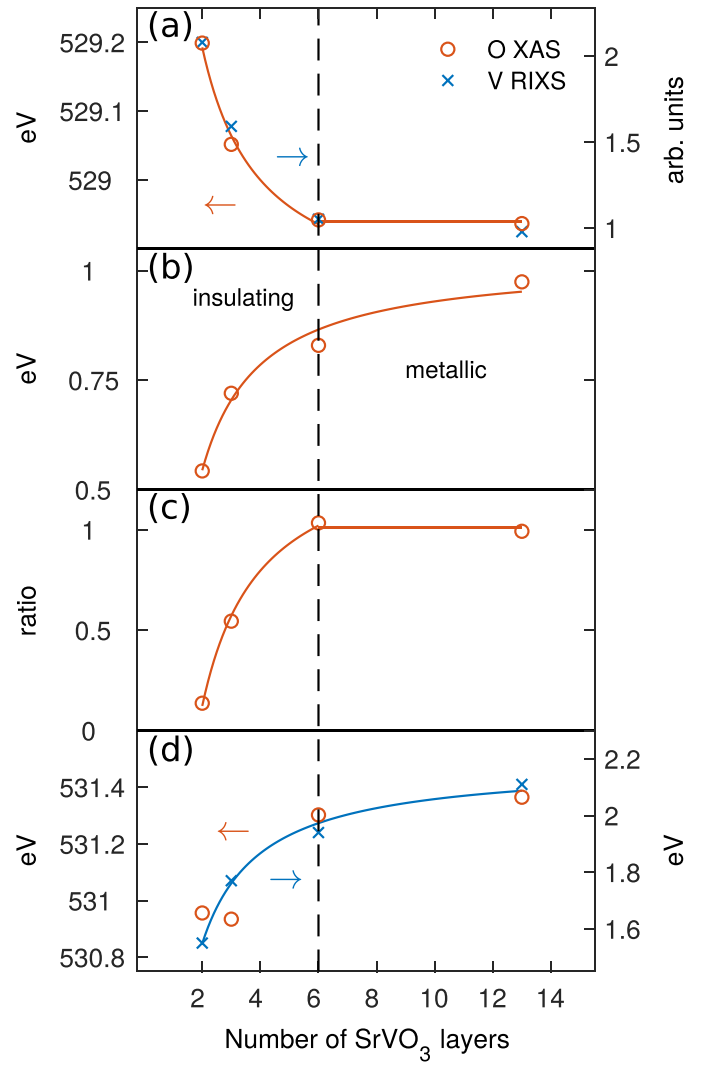

FIG. 5. Evolution of correlated electron behavior from experimental x-ray absorption spectroscopy (XAS) and resonant inelastic x-ray scattering (RIXS) measurements of $\mathrm{SrVO}_{3} / \mathrm{SrTiO}_{3}$ superlattices (SLs), reproduced from Ref. [5]. From top to bottom, evolution in (a) metallicity of SLs, (b) quasiparticle (QP) bandwidth, (c) QP spectral weight, and (d) the energy of the upper Hubbard band (UHB) are shown.

is directly observed, and its center is shown here. From V $L$ edge RIXS, the UHB energy is available from transitions from occupied QP states to the unoccupied UHB. Both show equivalent evolution with SL structure.

Figure 6 shows a schematic illustration comparing the extracted experimental quantities with their DFT + DMFT definitions. The QP bandwidth has been extracted from the DMFT spectral function by obtaining the width defined by the minima around the central QP peak. The QP ratio was determined by taking the ratio of the the $\mathrm{QP}$ weight (labeled $Q$ in Fig. 6) and the UHB weight (labeled $U$ ). Finally, the energy of the UHB was obtained by locating the peak in the DMFT spectral function, referenced to $\omega=0$. In the experimental RIXS process, the UHB peak energy represents the peak in the joint QP and UHB density of states and therefore is referenced to an energy $\omega<0$. To compare the theoretical and experimental UHB energies, we therefore shift all theoretical UHBs so that the 2:7 SLs match (the shift is $-0.584 \mathrm{eV}$ ).

\section{APPENDIX B: DFT CALCULATIONS}

DFT calculations were performed using the ELK full potential linearized augmented plane-wave (FP-LAPW) code within the LDA [31]. The results are in excellent agree- 


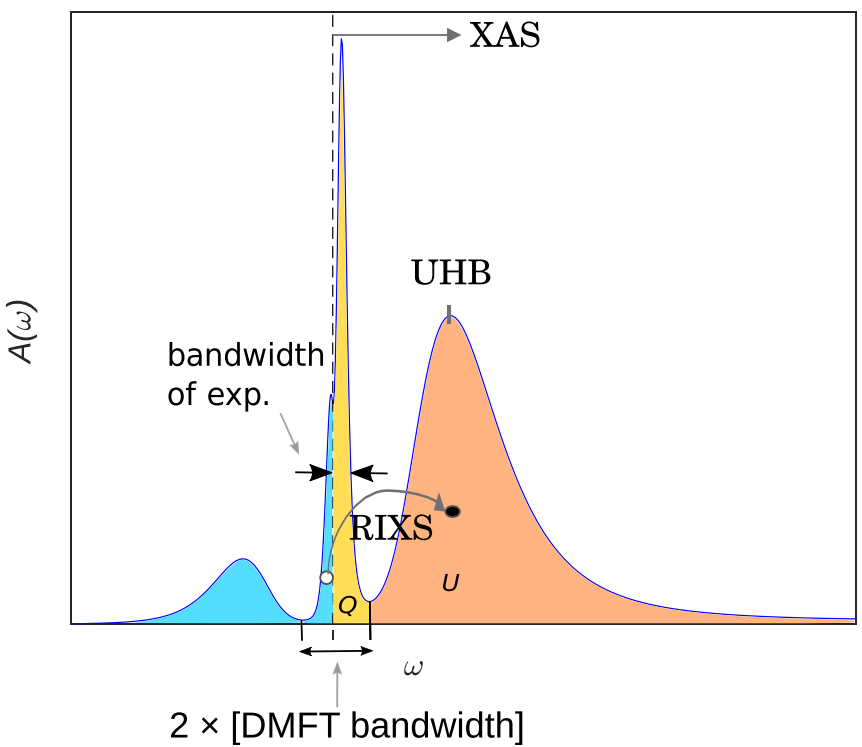

FIG. 6. A schematic illustration showing how the variables in the theory-experimental comparison were determined.

ment with previous pseudopotential calculations within the generalized gradient approximation (GGA) of the same SLs [5]. Previous photoemission spectroscopy work [17] shows how the dimensionality of $\mathrm{SrVO}_{3}$ influences the MIT. In their results, $10 \mathrm{SrVO}_{3}$ layers closely resemble bulk behavior. From this, we approximate the 13:4 SL in Ref. [5] with bulk DFT + DMFT calculations.

Self-consistency was achieved on a $12 \times 12 \times 4 k$-mesh in the full Brillouin zone (BZ) for relatively low computational cost with sufficient sampling, corresponding to $84 k$-points in the irreducible $\left(\frac{1}{16}\right)$ BZ. To stabilize the DFT self-consistent cycles, small values of mixing of the new potentials was used, at the cost of computational time. For bulk $\mathrm{SrVO}_{3}$, a $k$-mesh of $12 \times 12 \times 12$ was used ( $84 k$-points in the $\frac{1}{48}$ irreducible $\mathrm{BZ}$ ).

The partial densities of states (PDOS) of $t_{2 g}$ orbitals are shown in Fig. 7 for the bulk and 2:7, 3:6, and 6:5 SLs. Sharp peaks in the PDOS reflect the quantized electronic structure along $c$. For the inner layers of the thicker SLs, the PDOS more closely resembles that of bulk $\mathrm{SrVO}_{3}$, e.g., impurity 3 of the 6:5 SL. Near the interface, the $x z(y z)$ PDOS extends to higher energies as a result of mixing of these states with $\mathrm{Ti}$ states in the $\mathrm{SrTiO}_{3}$ layer.

The characters of the subbands are shown in Fig. 8 for each of the different $\mathrm{V}$ and Ti sites, using the 6:5 SL as an example. As expected, the $\mathrm{V}$ bands dominate the character at the Fermi level, with weak contribution from interfacial $\mathrm{Ti}$ ions. The spatial distribution of the subband wave functions of the $\mathrm{SrVO}_{3} \mathrm{QWs}$ can be seen directly in the characters. The $\mathrm{V} n=0$ subband, with greatest amplitude in the center of the well, has strong character in the central $\mathrm{V}$ ion and weak character at the interface. Correspondingly, the $\mathrm{V} n=2$ subband has the strongest character at the interface and is almost absent in the second layer close to where a node is expected in the QW wave function. At higher energies, the quantized $\mathrm{V} e_{g}$ subbands appear $>1 \mathrm{eV}$.
The Ti band characters shed light on the broadening of the Ti bands in the DFT + DMFT calculations shown in Fig. 3 . As above, the central $\mathrm{Ti}$ ion contributes strongly to the $\mathrm{Ti}$ $n=0$ and 2 subbands. On the other hand, the interfacial Ti ion contributes significantly to the Ti $n=1,2$, and 3 subbands, with the largest contribution to the Ti $n=2$ subband. The interfacial $\mathrm{Ti}$ ion mixes most strongly with the $\mathrm{V}$ orbitals, which are the correlated orbitals in the subsequent DMFT cycle. This demonstrates how the spatial penetration of the $\mathrm{Ti}$ $n=1$ and 2 subbands into the correlated $\mathrm{SrVO}_{3}$ layers leads to substantial broadening of these subbands in the subsequent DMFT cycle. In contrast, the Ti $n=0$ subband is spatially deep within the $\mathrm{SrTiO}_{3}$ layer and does not feel the effects of the correlated $\mathrm{SrVO}_{3}$ orbitals very strongly, remaining reasonably sharp even in the insulating phase [Fig. 3(j)].

\section{APPENDIX C: QTB MODEL}

\section{Bulk TB bands}

The TB model was constructed up to 12 th nearest neighbors, consisting of 24 hopping terms $t_{i}$ up to $[l, m, n]=$ $[2,2,2]$. For the $x y$ band, the TB dispersion $\varepsilon_{x y}$ is given by

$$
\varepsilon_{x y}=E_{x y}^{0}+\sum_{l m n} t_{x y}^{l m n} \cos \left(l k_{x}+m k_{y}+n k_{z}\right),
$$

where the band energy $E_{x y}^{0}$ corresponds to the CF energy. Since the purpose of our model is to accurately describe the bulk 3D DFT band structure, we do not attempt to analyze individual parameters, as has been done before [15]. Although terms corresponding to the fifth nearest neighbor and higher had a magnitude of $<10 \mathrm{meV}$, these terms were found to be necessary to adequately describe the FP-LAPW band structure. After fitting this model to the bulk LDA band structure in the full cubic BZ, we find the root-mean-square difference is $<11 \mathrm{meV}$, with a maximum difference of $70 \mathrm{meV}$.

\section{Quantum confinement}

To account for the effects of quantum confinement of the $\mathrm{V} 3 d$ electrons in the $\mathrm{SrVO}_{3}$ layers, we apply the BohrSommerfeld phase accumulation model [25]:

$$
2 k_{z}^{n}(E) L+\delta(E)=2 \pi n,
$$

where $n=0,1,2, \ldots$ is the quantum number, $2 k_{z}^{n}(E) L$ is the total phase accumulated in traveling through the $\mathrm{SrVO}_{3}$ layer and back, $k_{z}^{n}(E)$ is the quantized out-of-plane wave vector, $L=m c$ is the $\mathrm{SrVO}_{3}$ layer thickness ( $m$ and $c$ are the number of $\mathrm{SrVO}_{3}$ layers and $c$-axis lattice parameter of the $\mathrm{SrVO}_{3}$ layers, respectively), and $\delta(E)$ is the total phase acquired due to reflection at both $\mathrm{SrVO}_{3} / \mathrm{SrTiO}_{3}$ interfaces. For asymmetric QWs, e.g., thin overlayers with a vacuum interface, $\delta=\phi_{1}+\phi_{2}$ is composed of different individual phase shifts at each reflection; in our case of symmetric barriers, $\delta=2 \phi$, where $\phi$ is the phase at a single $\mathrm{SrVO}_{3} / \mathrm{SrTiO}_{3}$ interface. In general, $\delta=\delta(E)$ is explicitly dependent on the energy of the confined state. However, to simplify the fitting and avoid unnecessary degrees of freedom, we instead implicitly include the energy dependence through different phases for each quantum number $\delta=\delta_{n}$. With this, the quantization condition 

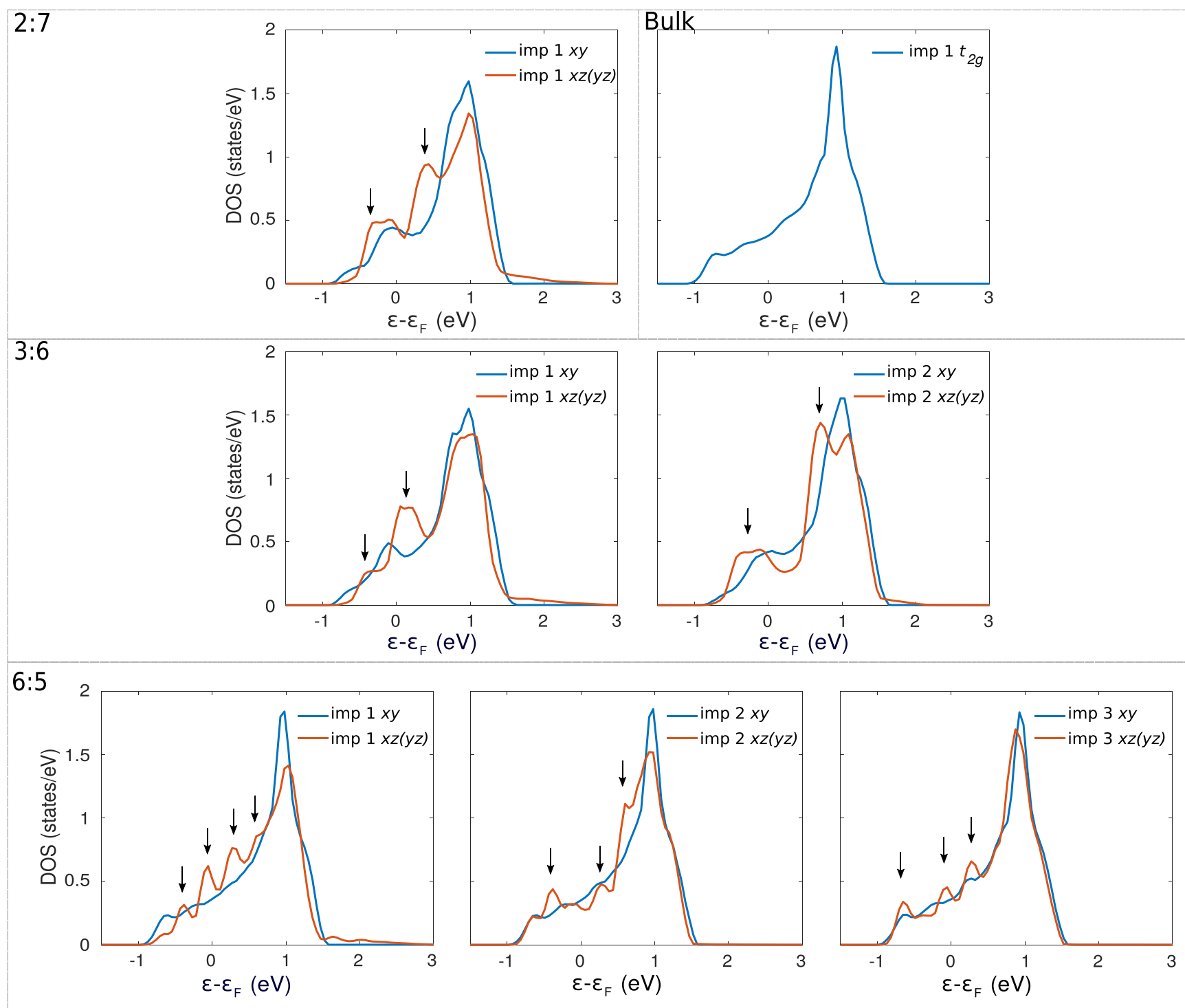

FIG. 7. The density functional theory (DFT) $\mathrm{V} t_{2 g}$ partial density of states (PDOS) of the bulk $\mathrm{SrVO}_{3}, 2: 7,3: 6$, and 6:5 SLs. The dashed borders outline which plots belong to the corresponding structure. Each panel shows the PDOS of the inequivalent $\mathrm{V}$ atoms in each structure, labeled by their impurity number in the dynamical mean-field theory (DMFT) cycles (imp 1 refers to the interface). The arrows indicate the contributions of quantized states to each inequivalent $\mathrm{V}$ atom. The greatest contribution is from the relatively flat bands along $\Gamma-X$.

reduces to

$$
k_{z}^{n}=\frac{2 \pi n-\delta_{n}}{2 m c}
$$

from which the quantized TB dispersion $E_{n}\left(k_{x}, k_{y}, k_{z}^{n}\right)$ may be evaluated.

\section{Full quantization parameters}

For each SL, four parameters were fitted to describe the intrinsic band structure, and $n$ parameters described the confined bands. The quantized TB dispersion was fitted to the FP-LAPW ELK band structure of the SLs. The four intrinsic parameters consist of band centers $\left[E_{i}^{0}\right.$ in Eq. (C1)] and band widths for the $x y$ and $x z(y z)$ bands. The band width parameter $W_{i}$ is a multiplicative factor to the hopping terms $t_{i}$ (the hopping terms themselves were fixed to the cubic bulk parameters determined above, effectively fixing the shape of the band). In addition to the intrinsic parameters, the phase shifts for each confined state $\delta_{n}$ were also fitted. The fitted phases are shown in Fig. 9 against the mean energy of each state and closely follow the same roughly linear relationship with energy for all SLs.

The results of fitting the FP-LAPW bands to the quantized TB model are shown in Table I, separated into contributions from the underlying bulk intrinsic bands and after quantizing these bands. An example of the fitted band structure is shown in Fig. 1 for the 2:7 SL. Since its wave function is perpendicular to the quantization axis, the $x y$ bandwidth is hardly affected by confinement, but the $x z(y z)$ bands are significantly narrowed compared with their intrinsic (bulklike) counterparts. The confinement leads to the preferential filling of the quantized $x z(y z)$ out-of-plane bands as their $k_{z}$ dispersion is 
V character
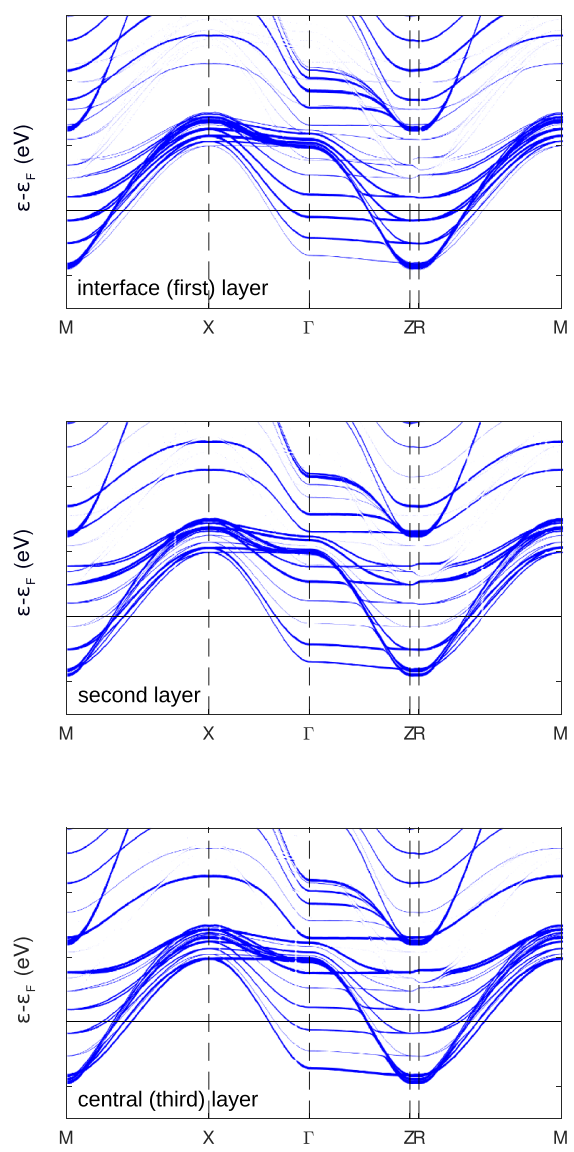

Ti character
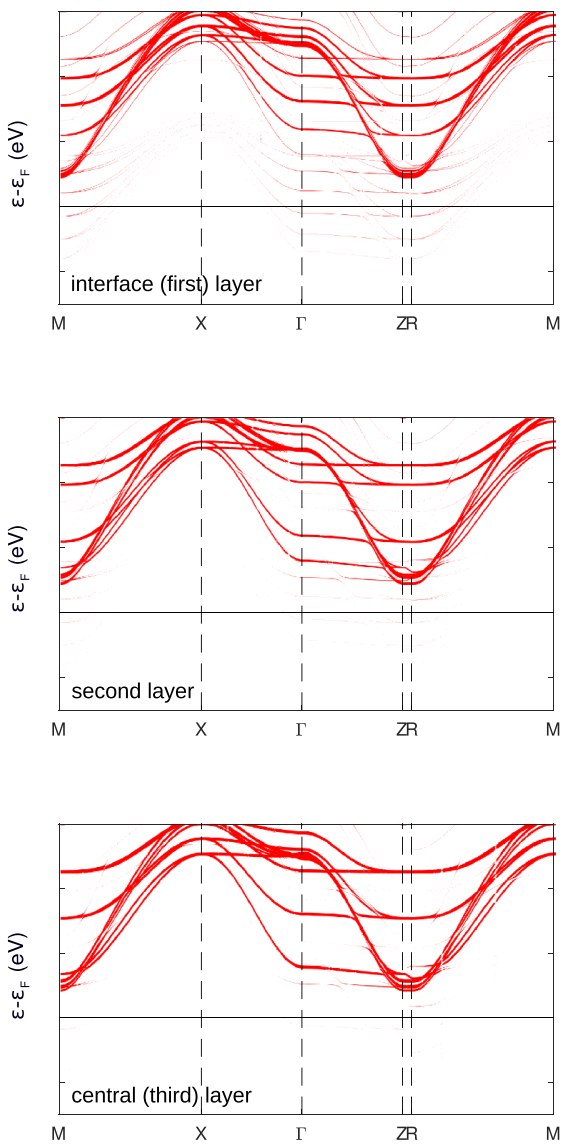

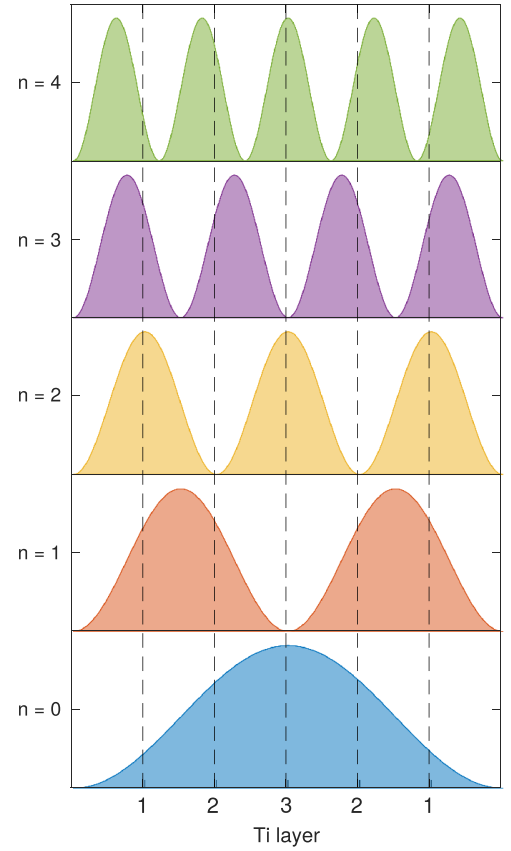

FIG. 8. The density functional theory (DFT) V and Ti $t_{2 g}$ band characters of the 6:5 superlattice (SL). The thickness of the lines indicates the total character of each $\mathrm{V}$ (left) and $\mathrm{Ti}$ (right) site. The top row shows the band characters at the interface, while the bottom row shows the character in the center of each layer. On the right, a schematic illustration of the real-space probability distribution of the quantized subbands in the out-of-plane direction of the $\mathrm{SrTiO}_{3}$ layers is shown. The edge of the box does not coincide with the interface Ti ion or its neighboring $\mathrm{SrO}$ layer due to the finite phase accumulated at the interface.

suppressed and they become 1D-like, which also pulls the Fermi level down slightly.

We note that confinement alone is capable of reproducing the SL band structure to a large extent, correctly describing the narrowing of the quantized bandwidth and its variation with $\mathrm{SrVO}_{3}$ layer thickness. This has been checked by restricting the intrinsic bands in the fit to the bulk bands (i.e., setting $E_{i}^{0}$

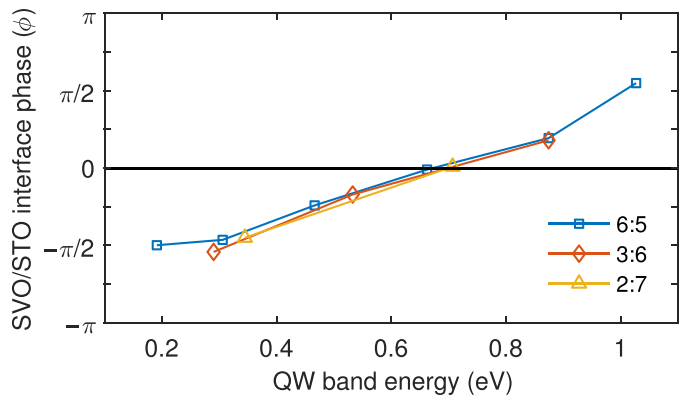

FIG. 9. The phase shift at the $\mathrm{SrVO}_{3} / \mathrm{SrTiO}_{3}$ interface for each quantized state in the superlattices (SLs; note $\phi_{n}=\delta_{n} / 2$ is shown), shown against the mean band energy. and $W_{i}$ to the bulk ones). This provides additional support that the band narrowing that eventually drives the MIT is primarily due to quantization effects rather than CF effects.

\section{APPENDIX D: DMFT CALCULATIONS}

The output from the ELK DFT calculation was imported to the TRIQS library [34] via an in-house interface with the DMFTPROJ [37] application. As in the literature [18,20,21],

TABLE I. Results of fitting the FP-LAPW ELK bands to a QTB model. The CF splitting is the energy difference $E_{y z}^{0}-E_{x y}^{0}$. The bandwidth (relative to bulk $\mathrm{SrVO}_{3}$ ) $W_{i}$ of the $x y$ and $y z$ bands is shown for both intrinsic bands (before quantization) and for the quantized bands, alongside their anisotropy $\left(W_{y z} / W_{x y}\right)$.

\begin{tabular}{|c|c|c|c|c|c|c|c|}
\hline \multirow[b]{2}{*}{ SL } & \multirow{2}{*}{$\begin{array}{c}\text { CF splitting } \\
(\mathrm{meV})\end{array}$} & \multicolumn{3}{|c|}{ Intrinsic } & \multicolumn{3}{|c|}{ Quantized bands } \\
\hline & & $W_{x y}$ & $W_{y z}$ & Anis. & $W_{x y}$ & $W_{y z}$ & Anis. \\
\hline $6: 5$ & 33 & 0.966 & 0.959 & 0.993 & 0.950 & 0.900 & 0.948 \\
\hline $3: 6$ & 40 & 0.971 & 0.960 & 0.989 & 0.935 & 0.798 & 0.8 \\
\hline $2: 7$ & 51 & 0.963 & 0.952 & 0.988 & 0.911 & 0.713 & 0.782 \\
\hline
\end{tabular}


only the $\mathrm{V} t_{2 g}$ bands were projected (using Wannier projectors) [35] to construct the LDA Hamiltonian in Wannier space. These projectors were constructed in the following correlated energy windows: $2: 7 \mathrm{SL},[-1.36,2.0] \mathrm{eV} ; 3: 6$ $\mathrm{SL},[-1.29,2.0] \mathrm{eV} ; 6: 5 \mathrm{SL},[-1.29,2.0] \mathrm{eV}$; and bulk, $[-1.50,1.90] \mathrm{eV}$. These windows were constructed such that all of the $\mathrm{V} \mathrm{t}_{2 g}$ bands are included, and the valence charge above the lower bound, corresponding to the charge in the $\mathrm{V}$ $t_{2 g}$ orbitals, is equal to 1 per V impurity. Each DMFT cycle calculation used $84 \times 10^{6}$ Monte Carlo sweeps.

To avoid potential complications from the ill-posed problem of analytic continuation, many quantities were determined from the Green's function and self-energy on the imaginary time $(\tau)$ or Matsubara frequency axis. The charge of each orbital $\left(n_{e}\right)$ was determined by

$$
n_{e}=\frac{1}{\beta} \sum_{n} G\left(i \omega_{n}\right) e^{i \omega_{n} 0^{+}},
$$

within the TRIQS library. As there is negligible interorbitalorbital overlap on the impurity, the $n_{e}$ orbital charge matrix is diagonal. The spectral function at the Fermi level is determined directly from the imaginary time Green's function by [18]

$$
A(\omega=0)=\frac{\beta G\left(\tau=\frac{1}{2} \beta\right)}{\pi},
$$

where $\beta$ is the inverse temperature. The $A(\omega=0)$ here is an averaged quantity over a frequency window $\sim \beta^{-1}$. The value of QP residue $Z$ was determined by

$$
Z=\left\{1-\left.\frac{\partial \operatorname{Im}\left[\Sigma\left(\mathrm{i} \omega_{n}\right)\right]}{\partial \mathrm{i} \omega_{n}}\right|_{\mathrm{i} \omega_{n} \rightarrow 0^{+}}\right\}^{-1},
$$

where the $Z$ is evaluated from the differential of the imaginary part of the Matsubara self-energy at i $\omega_{n} \rightarrow 0^{+}$. For $U$ values far from the Fermi liquid regime (namely, for the $6: 5 Z$ values close to the MIT), the $Z$ values were approximated by using the differential of the interpolated self-energies at $\mathrm{i} \omega_{n}=0$. There are two ways to realize the insulating solution: first, by a divergence in $\operatorname{Im}\left[\Sigma\left(\mathrm{i} \omega_{n}\right)\right]$, which comes naturally with $Z=0$. Second, the combination of the $\operatorname{Re}\left[\Sigma\left(\mathrm{i} \omega_{n}\right)\right]$ and the chemical potential might move the pole position outside of the noninteracting bandwidth, meaning that no QP peak is possible in the Green's function. In the latter case, we have $A(\omega=0)$ vanishing with nondiverging $\operatorname{Im}\left[\Sigma\left(\mathrm{i} \omega_{n}\right)\right]$. In that case that we also see here, we set $Z$ to zero manually. From this, the MIT $U$ value $\left(U_{\text {MIT }}\right)$ is defined as the lowest $U$ value in which $A(\omega=0)=0$.

The spectral functions $A(\omega)$ for each impurity were calculated from $G(\tau)$ using the LINEFITANALYZER technique of the maximum entropy analytic continuation method implemented within the MAXENT application of TRIQS [38]. The k-resolved spectral functions $A(\mathbf{k}, \omega)$ were calculated from the analytically continued self-energy.

The effective and correlation subband mass enhancement factors $1 / Z_{v}$ and $1 / Z_{v}^{\mathrm{c}}$ were calculated from the ratios of the Fermi velocities using

$$
Z_{v}^{\mathrm{c}}=\frac{v_{\mathrm{F}}^{c}}{v_{\mathrm{F}}^{\mathrm{QTB}}},
$$

and

$$
Z_{v}=\frac{v_{\mathrm{F}}^{c}}{v_{\mathrm{F}}^{i}}
$$

Here, the Fermi velocities were determined from the gradient of the linearly expanded band dispersions along $M-X$ around $k_{\mathrm{F}}$ of the DFT + DMFT subbands $\left(v_{\mathrm{F}}^{c}\right)$, the quantized bands from QTB $\left(v_{\mathrm{F}}^{\mathrm{QTB}}\right)$, and the intrinsic (bulklike) TB bands $\left(v_{\mathrm{F}}^{i}\right)$. The intrinsic bands were used, as they incorporate the effect of renormalization due to strain. Therefore, $Z_{v}^{\mathrm{c}}$ and $Z_{v}$ describe the effect of renormalization from correlations and the combination of correlations and confinement (band) effects, respectively.

The DFT + DMFT subband energy centers $E_{v, \mathbf{k}}$ were calculated by using

$$
E_{v, \mathbf{k}}=\epsilon_{v, \mathbf{k}}-\mu+\operatorname{Re}\left[\Sigma_{v}\left(\mathbf{k}, \omega=E_{v, \mathbf{k}}\right)\right],
$$

where $\epsilon_{v, \mathbf{k}}$ is the DFT energy, $\mu$ is the chemical potential, and $\operatorname{Re}\left[\Sigma_{v}(\mathbf{k}, \omega)\right]$ is the real part of the diagonal upfolded selfenergy elements on the real frequency axis. The QP lifetime in the inset of Fig. 4 was determined from the inverse imaginary part of the analytically continued upfolded self-energy. Finally, the subband energies at the $\Gamma$ high-symmetry point in Fig. 4 were determined from Eq. (D6).

\section{One-shot and fully charge self-consistent DFT + DMFT results}

The main text presents fully charge self-consistent (FCSC) DFT + DMFT calculations. Here, we present the results of one-shot (OS) DFT + DMFT for comparison. Overall, charge self-consistency slightly adjusts some details of the results, but the main conclusions of our paper are already present in OS calculations.

Figure 10 shows the $U$-dependent MIT for each SL and different DFT + DMFT methods. The behavior of the OS and FCSC calculations is very similar, exhibiting a $U_{\text {MIT }}$ with comparable characteristics, e.g., $A(\omega=0)$ and $Z$. Some differences are observed in the orbital polarization between the two methods, whereby the polarization is somewhat suppressed in the FCSC calculation compared with OS. This behavior, most notable for the 2:7 SL, is consistent with other studies [20-22] and is caused by the charge redistribution with the rest of the system at the DFT stage. This trend from 2:7 to bulk is also seen in Fig. 11 for the orbitally averaged values of $Z$, where there are some differences in $\bar{Z}$ for the 2:7 SL between OS and FCSC, but the bulk values are very similar.

An important note to make about Fig. 10 is that $Z$ at the interface (imp 1) for the $x z(y z)$ orbitals tends to zero first for each SL. This suggests that the weight from the $x z(y z) \mathrm{QP}$ peak depletes first. Therefore, when the interface $x z(y z) \mathrm{QP}$ state has been fully depleted, this causes the SL to transition into the insulating state. From this, the interface between the oxides has a strong influence on the MIT. The $A(\omega=0)$ for imp 1 of the $6: 5$ also tends to zero which strengthens the argument for at least that SL.

Figure 12 shows the $\mathrm{A}(\omega)$ of each correlated impurity orbital in each SL at $U=5.7 \mathrm{eV}$ (the value used in the theoretical-experimental comparisons). It is evident that the 2:7 is insulating and the $3: 6$ and 6:5 are metallic from the 


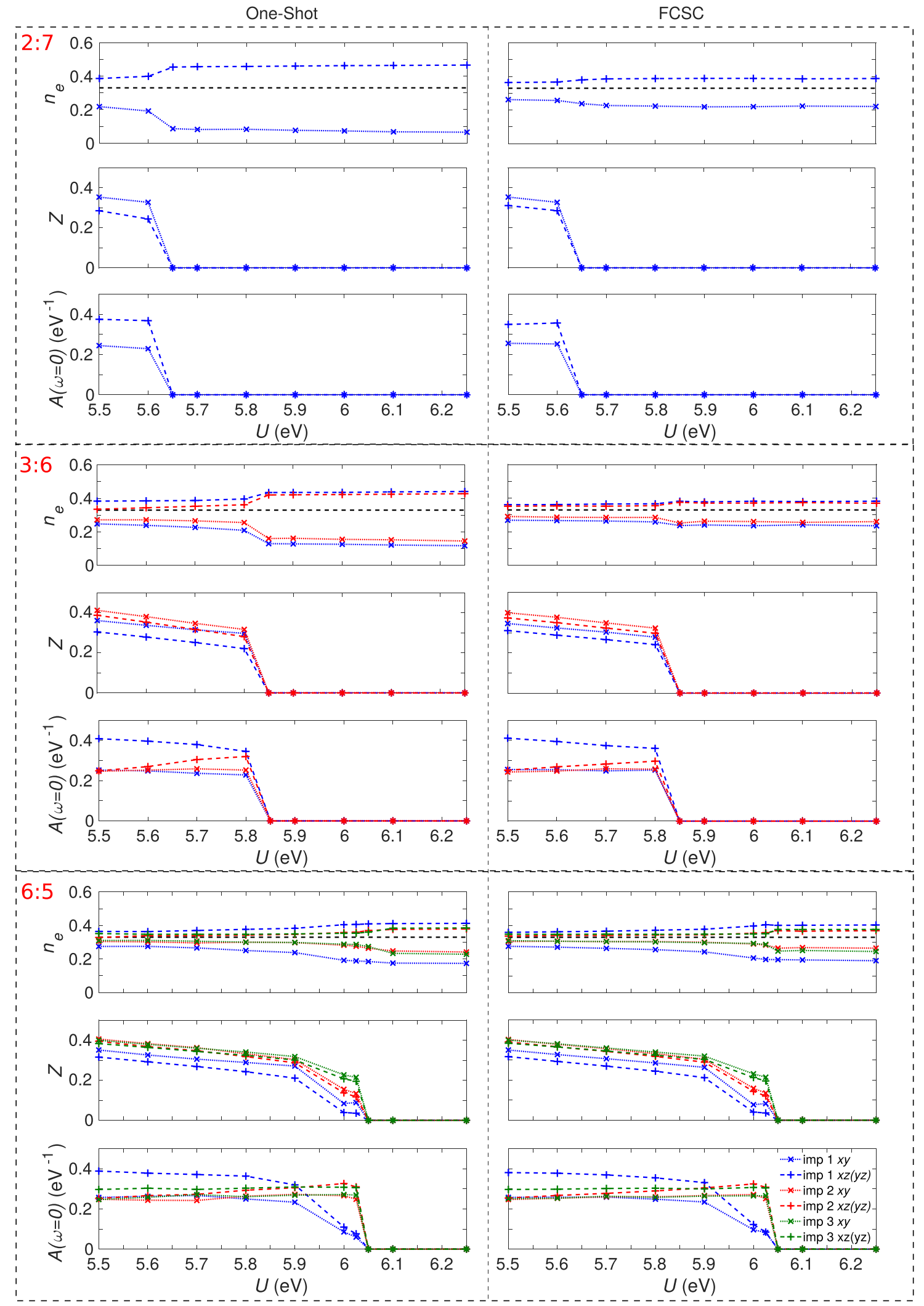

FIG. 10. The effect of $U$ on the orbital charge $n_{e}$ (top), quasiparticle (QP) residue $Z$ (middle), and the spectral function around the Fermi level (bottom) for each superlattice (SL) one-shot (OS) and fully charge self-consistent (FCSC) density functional theory (DFT) + dynamical mean-field theory (DMFT) calculation. These quantities were extracted from the corresponding Green's functions and self-energies on either the imaginary time or Matsubara frequency axis. The dashed line represents the bulk degenerate orbital charge. 


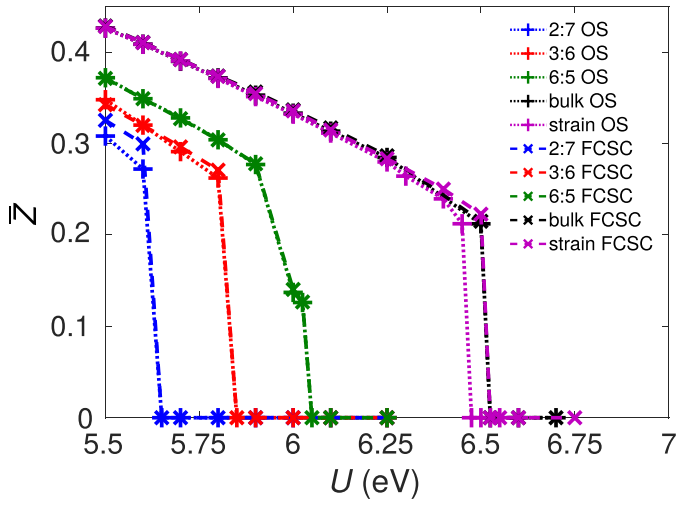

FIG. 11. The comparison of the orbitally averaged quasiparticle (QP) residue $\bar{Z}$ between the one-shot (OS) and fully charge selfconsistent (FCSC) density functional theory (DFT) + dynamical mean-field theory (DMFT) methods. The plot lines are guides to the eye.

absence or presence of the QP peak at the Fermi level. There are sharp features in the QP peaks around the Fermi level for the 3:6 and 6:5 SLs. These features are often attributed to spurious noise from the analytic continuation procedure; however, it may not be the case here due to the quantized bands being present around the Fermi level. The peak position of the Hubbard bands (notably the UHB) are closer in energy to the Fermi level for the interface layer (impurity 1) than the other layers for the 3:6 and 6:5. This is another indication that the interface layer is more correlated than the other layers.

The trends across the SLs give further insight into the physical phenomena. The splitting of the orbital degeneracy strongly affects the polarization of the orbital charge, which is shown by the averaged Wannier orbital charge in Fig. 13. The reduction of the number of layers significantly increases

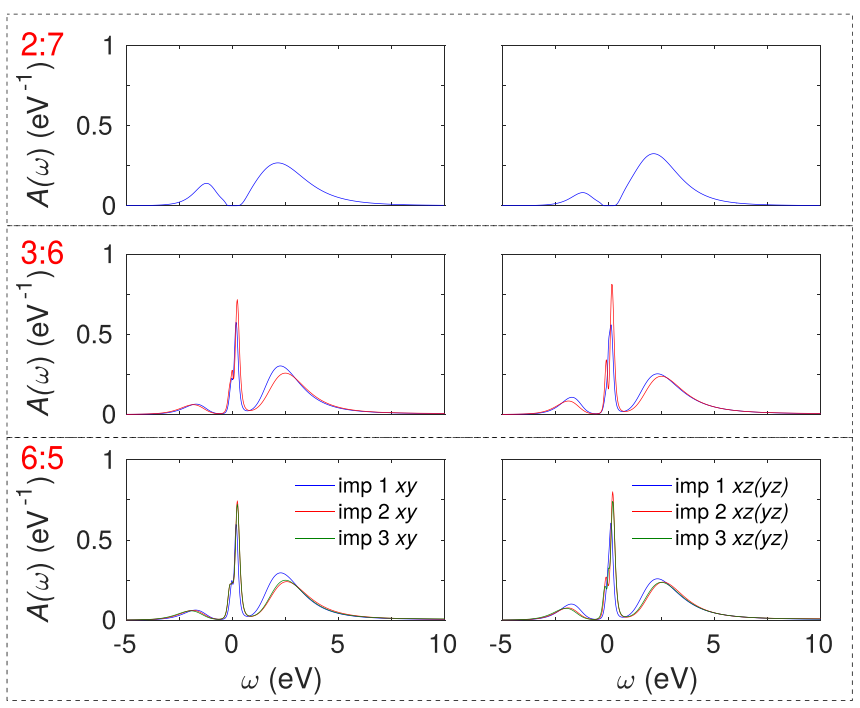

FIG. 12. Spectral functions of the 2:7 (top), 3:6 (middle), and 6:5 (bottom) superlattices (SLs) from fully charge self-consistent (FCSC) calculations, showing $x y$ (left) and $x z(y z)$ (right) orbitals. The spectra of the insulating 2:7 SL have been shifted such that the Fermi level lies at the center of the band gap of the $x y$ spectrum.

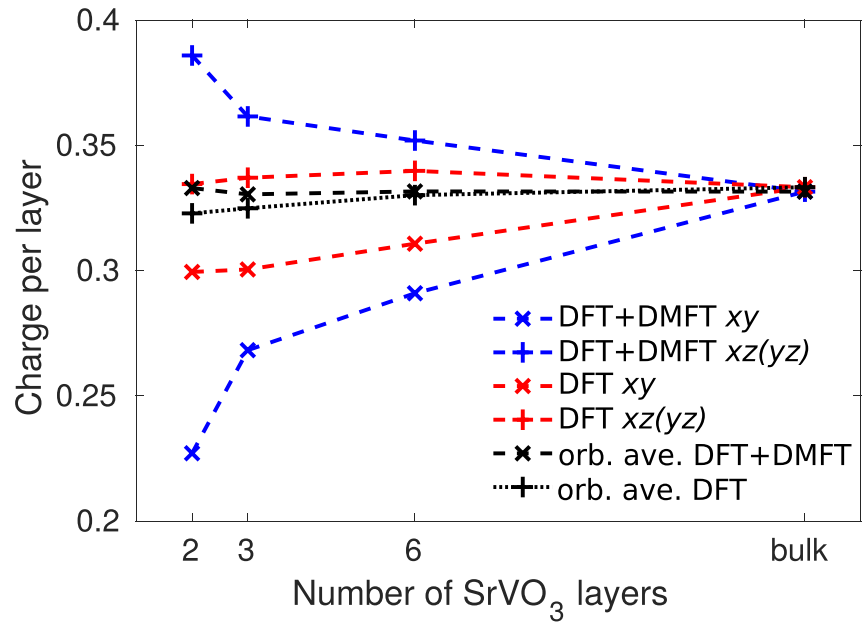

FIG. 13. The averaged orbital charge over all layers from the density functional theory (DFT) and DFT + dynamical mean-field theory (DMFT) Wannier V $x y$ and $x z(y z)$ orbitals for each superlattice (SL) and bulk. This includes charges from DFT, DFT + DMFT, and average orbital charge per layer (orb. ave.) for each SL and bulk.

the charge in the $x z(y z)$ orbitals, which appear to tend to half-filling (whereas the $x y$ orbitals are tending towards zero charge). This is a likely consequence of these orbitals trying to reduce the potential energy, analogous to what is seen in the previous monolayer calculations.

Interestingly, the DFT averaged V Wanner orbital charge over all $\mathrm{SrVO}_{3}$ layers for the decreasing number of $\mathrm{SrVO}_{3}$ SLs is slightly (but noteworthy) lower than $\frac{1}{3}$ (the sum of all of these averaged Wanner $t_{2 g}$ orbitals is equal to $\sim 1$, as expected). The Ti states have a small amount of charge, as shown in Table II. This table shows the DFT muffin tin (of each species) and interstitial charge percentages calculated from integrated DOS within the correlated energy windows (used for the DMFT calculations). The total charge equals the number of $\mathrm{V}$ atoms (which each contribute one valence electron) in the unit cell (i.e., for the 3:6 SL, the total charge in the correlated energy window is three). As can be seen in Table II, the Ti charge percentage contribution decreases with increasing number of $\mathrm{SrVO}_{3}$ layers. The greatest contribution to the Ti charge is from the interface $\mathrm{Ti}$ [3d $x z(y z)$ states], shown in Table II. The difference in the total and interface $\mathrm{Ti}$ contributions corresponds to the charge in the other $\mathrm{SrTiO}_{3}$ layers, which is small compared with the interface Ti charge. Therefore, the charge is likely to be primarily leaking from

TABLE II. The DFT integrated DOS percentages (\%) of the total charge within the correlated energy window. The charge per interface $\mathrm{Ti}$ atom is given to show how much charge has leaked to the Ti interface.

\begin{tabular}{lcccccc}
\hline \hline SL & Interstitial & $\mathrm{Sr}$ & $\mathrm{V}$ & $\mathrm{O}$ & $\mathrm{Ti}$ & Ti interface \\
\hline $2: 7$ & 19.1 & 0.6 & 66.6 & 10.8 & 2.9 & $2.7(=0.027 \mathrm{e}-/ \mathrm{Ti})$ \\
$3: 6$ & 18.9 & 0.6 & 67.3 & 10.9 & 2.3 & $2.1(=0.0315 \mathrm{e}-/ \mathrm{Ti})$ \\
$6: 5$ & 18.9 & 0.6 & 68.5 & 10.8 & 1.2 & $1.1(=0.033 \mathrm{e}-/ \mathrm{Ti})$ \\
Bulk & 19.2 & 0.7 & 69.1 & 11.0 & - & - \\
\hline \hline
\end{tabular}



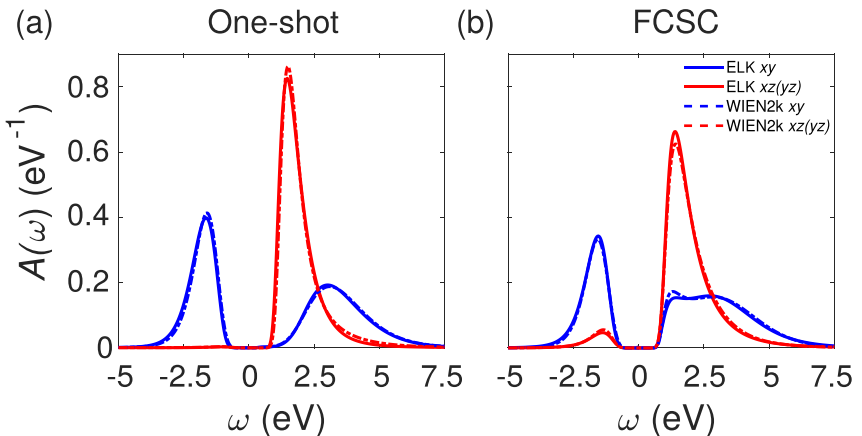

FIG. 14. The orbital $\mathrm{A}(\omega)$ comparisons between the one-shot (OS) and fully charge self-consistent (FCSC) density functional theory $(\mathrm{DFT})+$ dynamical mean-field theory (DMFT) methods from different input DFT codes for monolayer $\mathrm{SrVO}_{3}$.

the $\mathrm{V}$ to the interface $\mathrm{Ti}$ atoms (due to hybridization between the $\mathrm{V} t_{2 g}$ and $\mathrm{Ti} t_{2 g}$ at the interface), as indicated by the increasing $\mathrm{V}$ charge contribution with the number of $\mathrm{SrVO}_{3}$ layers. However, the amount of charge on the (interface) $\mathrm{Ti}$ atoms is small and only a few percent of the total charge (consistent with the small DFT Wanner charge deviation in Fig. 13), which is quite small. For this reason, the Ti states were not treated within DMFT.

\section{Effects of strain}

We performed volume-conserving strain calculations on bulk $\mathrm{SrVO}_{3}$ to investigate the effect CF has on the MIT while the bandwidths of the $t_{2 g}$ orbitals are approximately unchanged. Compressive strain of $1 \%$ was applied along the $c$ axis; the other axes were tensively strained to conserve volume compared with the bulk. This strain was chosen to yield a CF splitting of $53 \mathrm{meV}$, slightly larger than but comparable with the CF splitting of the 2:7 SL. The strained FCSC $U_{\text {MIT }}$ is $\sim 6.525 \mathrm{eV}$, the same as for the bulk. The OS strained calculation had a slightly lower $U_{\text {MIT }}$ of $6.475 \mathrm{eV}$. Due to the small change on $U_{\mathrm{MIT}}$, the CF splitting is insufficient to cause the MIT in these SLs.

\section{Comparing with the monolayer}

The results presented used an in-house interface between ELK and TRIQS, so this section presents comparison results between ELK and WIEN2K inputs into TRIQS to show that the interface works for a similar system, namely, monolayer $\mathrm{SrVO}_{3}$. The monolayer $\mathrm{SrVO}_{3}$ calculation was set up in the same way and using the same parameters as in Ref. [21]. Figure 14 shows the comparison between $A(\omega)$ calculated

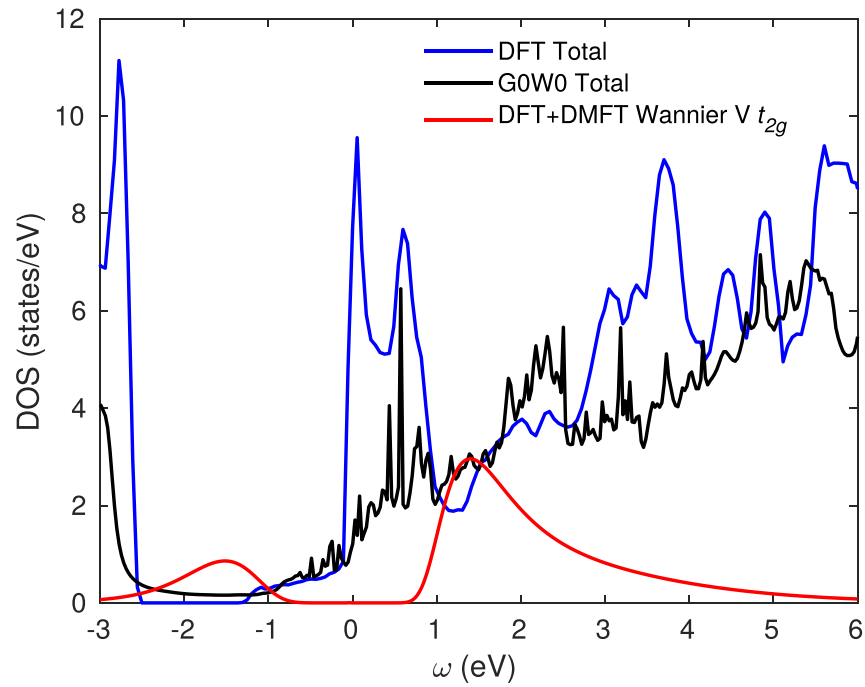

FIG. 15. Comparison of the density functional theory (DFT) total density of states (DOS), one-shot (OS) $G W(G 0 W 0)$ total spectral function and the fully charge self-consistent (FCSC) DFT + dynamical mean-field theory (DMFT) Wannier $\mathrm{V} t_{2 g}$ spectral function.

from the different DFT code inputs. This comparison shows excellent agreement between the different inputs for the different DFT + DMFT methods. This test shows that the interface used between ELK and TRIQS is able to reliably perform DFT + DMFT calculations.

To show that the insulating phase in these heterostructures is a strongly correlated effect, we performed a OS $G W$ $(G 0 W 0)$ calculation on the free standing $\mathrm{SrVO}_{3}$ monolayer within ELK to see if the insulating state can be obtained by including nonlocal exchange effects. The electron correlations in the monolayer (originating from dimensionality effects here) are expected to be the greatest out of all systems studied in this paper. The $G 0 W 0$ calculation started from the converged monolayer DFT calculation and used a $k$-mesh of $15 \times 15 \times 1, q$-mesh of $5 \times 5 \times 1$, maximum Matsubara frequency cutoff of $272.11 \mathrm{eV}$, and $\beta=7.74 \mathrm{eV}^{-1}$ (note this high temperature will notably smear the spectral function). Figure 15 shows that both DFT and $G 0 W 0$ predict the system to be metallic, whereas the DFT + DMFT results are insulating, which is in line with the experimental results. The more subtle sharp features seen in the DFT have likely smeared out in $G 0 W 0$, but the noisy features in the $G 0 W 0$ spectral function are likely from the BZ integration (which did not use interpolation; BZ integrations with interpolation cannot be used with nonlocal quantities). The metallic $G 0 W 0$ results show that the experimentally observed insulating state in the heterostructures is most likely due to the strong electron correlations, as described by DFT + DMFT.
[1] Y. Tokura, M. Kawasaki, and N. Nagaosa, Emergent functions of quantum materials, Nat. Phys. 13, 1056 (2017).

[2] M. Brahlek, L. Zhang, J. Lapano, H.-T. Zhang, R. EngelHerbert, N. Shukla, S. Datta, H. Paik, and D. G. Schlom, Opportunities in vanadium-based strongly correlated electron systems, MRS Commun. 7, 27 (2017); J. Mannhart and D. G. Schlom, Oxide interfaces-an opportunity for electronics, Science 327, 1607 (2010).

[3] G. Kotliar, S. Y. Savrasov, K. Haule, V. S. Oudovenko, O. Parcollet, and C. A. Marianetti, Electronic structure calculations 
with dynamical mean-field theory, Rev. Mod. Phys. 78, 865 (2006).

[4] M. Izumi, Y. Ogimoto, Y. Konishi, T. Manako, M. Kawasaki, and Y. Tokura, Perovskite superlattices as tailored materials of correlated electrons, Mater. Sci. Eng. B 84, 53 (2001); A. Ohtomo and H. Y. Hwang, A high-mobility electron gas at the $\mathrm{LaAlO}_{3} / \mathrm{SrTiO}_{3}$ heterointerface, Nature (London) 427, 423 (2004); H. Y. Hwang, Y. Iwasa, M. Kawasaki, B. Keimer, N. Nagaosa, and Y. Tokura, Emergent phenomena at oxide interfaces, Nat. Mater. 11, 103 (2012); J. Chakhalian, J. W. Freeland, A. J. Millis, C. Panagopoulos, and J. M. Rondinelli, Colloquium: emergent properties in plane view: strong correlations at oxide interfaces, Rev. Mod. Phys. 86, 1189 (2014).

[5] J. Laverock, M. Gu, V. Jovic, J. W. Lu, S. A. Wolf, R. M. Qiao, W. Yang, and K. E. Smith, Nano-engineering of electron correlation in oxide superlattices, Nano Futures 1, 031001 (2017).

[6] I. A. Nekrasov, G. Keller, D. E. Kondakov, A. V. Kozhevnikov, Th. Pruschke, K. Held, D. Vollhardt, and V. I. Anisimov, Comparative study of correlation effects in $\mathrm{CaVO}_{3}$ and $\mathrm{SrVO}_{3}$, Phys. Rev. B 72, 155106 (2005).

[7] T. Yoshida, M. Hashimoto, T. Takizawa, A. Fujimori, M. Kubota, K. Ono, and H. Eisaki, Mass renormalization in the bandwidth-controlled Mott-Hubbard systems $\mathrm{SrVO}_{3}$ and $\mathrm{CaVO}_{3}$ studied by angle-resolved photoemission spectroscopy, Phys. Rev. B 82, 085119 (2010); S. Aizaki, T. Yoshida, K. Yoshimatsu, M. Takizawa, M. Minohara, S. Ideta, A. Fujimori, K. Gupta, P. Mahadevan, K. Horiba, H. Kumigashira, and M. Oshima, Self-Energy on the Low- to High-Energy Electronic Structure of Correlated Metal $\mathrm{SrVO}_{3}$, Phys. Rev. Lett. 109, 056401 (2012).

[8] A. Sekiyama, H. Fujiwara, S. Imada, S. Suga, H. Eisaki, S. I. Uchida, K. Takegahara, H. Harima, Y. Saitoh, I. A. Nekrasov, G. Keller, D. E. Kondakov, A. V. Kozhevnikov, Th. Pruschke, K. Held, D. Vollhardt, and V. I. Anisimov, Mutual Experimental and Theoretical Validation of Bulk Photoemission Spectra of $\mathrm{Sr}_{1-x} \mathrm{Ca}_{x} \mathrm{VO}_{3}$, Phys. Rev. Lett. 93, 156402 (2004).

[9] H. F. Pen, M. Abbate, A. Fujimori, Y. Tokura, H. Eisaki, S. Uchida, and G. A. Sawatzky, Electronic structure of $\mathrm{Y}_{1-x} \mathrm{Ca}_{x} \mathrm{VO}_{3}$ studied by high-energy spectroscopies, Phys. Rev. B 59, 7422 (1999); J. Laverock, B. Chen, K. E. Smith, R. P. Singh, G. Balakrishnan, M. Gu, J. W. Lu, S. A. Wolf, R. M. Qiao, W. Yang, and J. Adell, Resonant Soft-X-Ray Emission as a Bulk Probe of Correlated Electron Behavior in Metallic $\mathrm{Sr}_{x} \mathrm{Ca}_{1-x} \mathrm{VO}_{3}$, Phys. Rev. Lett. 111, 047402 (2013).

[10] A. Georges, G. Kotliar, W. Krauth, and M. J. Rozenberg, Dynamical mean-field theory of strongly correlated fermion systems and the limit of infinite dimensions, Rev. Mod. Phys. 68, 13 (1996).

[11] I. A. Nekrasov, K. Held, G. Keller, D. E. Kondakov, Th. Pruschke, M. Kollar, O. K. Andersen, V. I. Anisimov, and D. Vollhardt, Momentum-resolved spectral functions of $\mathrm{SrVO}_{3}$ calculated by LDA + DMFT, Phys. Rev. B 73, 155112 (2006).

[12] K. Byczuk, M. Kollar, K. Held, Y.-F. Yang, I. A. Nekrasov, Th. Pruschke, and D. Vollhardt, Kinks in the dispersion of strongly correlated electrons, Nat. Phys. 3, 168 (2007); J. M. Tomczak, M. Casula, T. Miyake, F. Aryasetiawan, and S. Biermann, Combined GW and dynamical mean-field theory: dynamical screening effects in transition metal oxides, Europhys. Lett. 100, 67001 (2012).
[13] L. Hedin, New method for calculating the one-particle Green's function with application to the electron-gas problem, Phys. Rev. 139, A796 (1965).

[14] L. Boehnke, F. Nilsson, F. Aryasetiawan, and P. Werner, When strong correlations become weak: consistent merging of $G W$ and DMFT, Phys. Rev. B 94, 201106(R) (2016).

[15] K. Maiti, D. D. Sarma, M. J. Rozenberg, I. H. Inoue, H. Makino, O. Goto, M. Pedio, and R. Cimino, Electronic structure of $\mathrm{Ca}_{1-x} \mathrm{Sr}_{x} \mathrm{VO}_{3}$ : A tale of two energy scales, Europhys. Lett. 55, 246 (2001); A. Liebsch, Surface versus Bulk Coulomb Correlations in Photoemission Spectra of $\mathrm{SrVO}_{3}$ and $\mathrm{CaVO}_{3}$, Phys. Rev. Lett. 90, 096401 (2003); J. Laverock, J. Kuyyalil, B. Chen, R. P. Singh, B. Karlin, J. C. Woicik, G. Balakrishnan, and K. E. Smith, Enhanced electron correlations at the $\mathrm{Sr}_{x} \mathrm{Ca}_{1-x} \mathrm{VO}_{3}$ surface, Phys. Rev. B 91, 165123 (2015).

[16] H. Ishida, D. Wortmann, and A. Liebsch, Electronic structure of $\mathrm{SrVO}_{3}(001)$ surfaces: a local-density approximation plus dynamical mean-field theory calculation, Phys. Rev. B 73, 245421 (2006).

[17] K. Yoshimatsu, T. Okabe, H. Kumigashira, S. Okamoto, S. Aizaki, A. Fujimori, and M. Oshima, Dimensional-CrossoverDriven Metal-Insulator Transition in $\mathrm{SrVO}_{3}$ Ultrathin Films, Phys. Rev. Lett. 104, 147601 (2010).

[18] Z. Zhong, M. Wallerberger, J. M. Tomczak, C. Taranto, N. Parragh, A. Toschi, G. Sangiovanni, and K. Held, Electronics with Correlated Oxides: $\mathrm{SrVO}_{3} / \mathrm{SrTiO}_{3}$ as a Mott Transistor, Phys. Rev. Lett. 114, 246401 (2015).

[19] M. Gu, S. A. Wolf, and J. Lu, Two-dimensional Mott insulators in $\mathrm{SrVO}_{3}$ ultrathin films, Adv. Mater. Interfaces 1, 1300126 (2014).

[20] S. Bhandary, E. Assmann, M. Aichhorn, and K. Held, Charge self-consistency in density functional theory combined with dynamical mean field theory: $k$-space reoccupation and orbital order, Phys. Rev. B 94, 155131 (2016).

[21] M. Schüler, O. E. Peil, G. J. Kraberger, R. Pordzik, M. Marsman, G. Kresse, T. O. Wehling, and M. Aichhorn, Charge self-consistent many-body corrections using optimized projected localized orbitals, J. Phys.: Conden. Matter 30, 475901 (2018).

[22] A. Hampel, S. Beck, and C. Ederer, Effect of charge self-consistency in DFT + DMFT calculations for complex transition metal oxides, Phys. Rev. Res. 2, 033088 (2020).

[23] S. Beck, G. Sclauzero, U. Chopra, and C. Ederer, Metalinsulator transition in $\mathrm{CaVO}_{3}$ thin films: interplay between epitaxial strain, dimensional confinement, and surface effects, Phys. Rev. B 97, 075107 (2018); G. Sclauzero, K. Dymkowski, and C. Ederer, Tuning the metal-insulator transition in $d^{1}$ and $d^{2}$ perovskites by epitaxial strain: a first-principles-based study, ibid. 94, 245109 (2016).

[24] L. Colakerol, T. D. Veal, H.-K. Jeong, L. Plucinski, A. DeMasi, T. Learmonth, P.-A. Glans, S. Wang, Y. Zhang, L. F. J. Piper, P. H. Jefferson, A. Fedorov, T.-C. Chen, T. D. Moustakas, C. F. McConville, and K. E. Smith, Quantized Electron Accumulation States in Indium Nitride Studied by Angle-Resolved Photoemission Spectroscopy, Phys. Rev. Lett. 97, 237601 (2006).

[25] T.-C. Chiang, Photoemission studies of quantum well states in thin films, Surf. Sci. Rep. 39, 181 (2000). 
[26] J. Liu, M. Kareev, D. Meyers, B. Gray, P. Ryan, J. W. Freeland, and J. Chakhalian, Metal-Insulator Transition and Orbital Reconstruction in Mott-Type Quantum Wells Made of $\mathrm{NdNiO}_{3}$, Phys. Rev. Lett. 109, 107402 (2012); S. G. Jeong, T. Min, S. Woo, J. Kim, Y.-Q. Zhang, S. W. Cho, J. Son, Y.-M. Kim, J. H. Han, S. Park, H. Y. Jeong, H. Ohta, S. Lee, T. W. Noh, J. Lee, and W. S. Choi, Phase Instability amid Dimensional Crossover in Artificial Oxide Crystal, ibid. 124, 026401 (2020).

[27] J. K. Kawasaki, C. H. Kim, J. N. Nelson, S. Crisp, C. J. Zollner, E. Biegenwald, J. T. Heron, C. J. Fennie, D. G. Schlom, and K. M. Shen, Engineering Carrier Effective Masses in Ultrathin Quantum Wells of $\mathrm{IrO}_{2}$, Phys. Rev. Lett. 121, 176802 (2018).

[28] K. Yoshimatsu, K. Horiba, H. Kumigashira, T. Yoshida, A. Fujimori, and M. Oshima, Metallic quantum well states in artificial structures of strongly correlated oxide, Science 333, 319 (2011).

[29] M. Kobayashi, K. Yoshimatsu, E. Sakai, M. Kitamura, K. Horiba, A. Fujimori, and H. Kumigashira, Origin of the Anomalous Mass Renormalization in Metallic Quantum Well States of Strongly Correlated Oxide $\mathrm{SrVO}_{3}$, Phys. Rev. Lett. 115, 076801 (2015).

[30] R. Adler, C.-J. Kang, C.-H. Yee, and G. Kotliar, Correlated materials design: prospects and challenges, Rep. Prog. Phys. 82, 012504 (2019).

[31] J. K. Dewhurst, S. Sharma, L. Nordström, F. Cricchio, O. Granas, and E. K. U. Gross, http://elk.sourceforge.net/.

[32] M. Gu, S. A. Wolf, and J. Lu, Transport phenomena in $\mathrm{SrVO}_{3} / \mathrm{SrTiO}_{3}$ superlattices, J. Phys. D: Appl. Phys. 51, 10LT01 (2018).

[33] P. Seth, I. Krivenko, M. Ferrero, and O. Parcollet, TRIQS/CTHYB: A continuous-time quantum Monte Carlo hybridisation expansion solver for quantum impurity problems, Comput. Phys. Commun. 200, 274 (2016).

[34] O. Parcollet, M. Ferrero, T. Ayral, H. Hafermann, I. Krivenko, L. Messio, and P. Seth, TRIQS: A toolbox for research on interacting quantum systems, Comput. Phys. Commun. 196, 398 (2015).

[35] M. Aichhorn, L. Pourovskii, V. Vildosola, M. Ferrero, O. Parcollet, T. Miyake, A. Georges, and S. Biermann, Dynamical mean-field theory within an augmented plane-wave framework: assessing electronic correlations in the iron pnictide LaFeAsO, Phys. Rev. B 80, 085101 (2009).

[36] F. Lechermann, Oxide Heterostructures from a Realistic Many-Body Perspective, in Handbook of Materials Modeling: Applications: Current and Emerging Materials, edited by W. Andreoni and S. Yip (Springer International Publishing, Cham, 2018), pp. 1-20.
[37] M. Aichhorn, L. Pourovskii, and A. Georges, Importance of electronic correlations for structural and magnetic properties of the iron pnictide superconductor LaFeAsO, Phys. Rev. B 84, 054529 (2011); M. Aichhorn, L. Pourovskii, P. Seth, V. Vildosola, M. Zingl, O. E. Peil, X. Deng, J. Mravlje, G. J. Kraberger, C. Martins, M. Ferrero, and O. Parcollet, TRIQS/DFTTools: a TRIQS application for $a b$ initio calculations of correlated materials, Comput. Phys. Commun. 204, 200 (2016).

[38] G. J. Kraberger, R. Triebl, M. Zingl, and M. Aichhorn, Maximum entropy formalism for the analytic continuation of matrix-valued Green's functions, Phys. Rev. B 96, 155128 (2017).

[39] S. Bhandary and K. Held, Self-energy self-consistent density functional theory plus dynamical mean field theory, arXiv:1904.02967v1.

[40] J. Laverock, M. Gu, V. Jovic, J. W. Lu, S. A. Wolf, G. Watson, R. M. Qiao, W. Yang, and K. E. Smith, Electronic reconstructions at the interface of $\mathrm{SrVO}_{3} / \mathrm{SrTiO}_{3}$ superlattices, unpublished (2020).

[41] S. Okamoto, Anomalous mass enhancement in strongly correlated quantum wells, Phys. Rev. B 84, 201305(R) (2011).

[42] H. Oka, Y. Okada, T. Hitosugi, and T. Fukumura, Two distinct surface terminations of $\mathrm{SrVO}_{3}(001)$ ultrathin films as an influential factor on metallicity, Appl. Phys. Lett. 113, 171601 (2018); G. Wang, Z. Wang, M. Meng, M. Saghayezhian, L. Chen, C. Chen, H. Guo, Y. Zhu, E. W. Plummer, and J. Zhang, Role of disorder and correlations in the metal-insulator transition in ultrathin $\mathrm{SrVO}_{3}$ films, Phys. Rev. B 100, 155114 (2019).

[43] I. Matsuda, T. Ohta, and H. W. Yeom, In-plane dispersion of the quantum-well states of the epitaxial silver films on silicon, Phys. Rev. B 65, 085327 (2002); K. Yoshimatsu, E. Sakai, M. Kobayashi, K. Horiba, T. Yoshida, A. Fujimori, M. Oshima, and $\mathrm{H}$. Kumigashira, Determination of the surface and interface phase shifts in metallic quantum well structures of perovskite oxides, ibid. 88, 115308 (2013).

[44] C. H. Ahn, S. Gariglio, P. Paruch, T. Tybell, L. Antognazza, and J.-M. Triscone, Electrostatic modulation of superconductivity in ultrathin $\mathrm{GdBa}_{2} \mathrm{Cu}_{3} \mathrm{O}_{7-x}$ films, Science 284, 1152 (1999).

[45] H. Chen, A. J. Millis, and C. A. Marianetti, Engineering Correlation Effects via Artificially Designed Oxide Superlattices, Phys. Rev. Lett. 111, 116403 (2013).

[46] S. Samanta, S. B. Mishra, and B. R. K. Nanda, Quantum well structure of a double perovskite superlattice and formation of a spin-polarized two-dimensional electron gas, Phys. Rev. B 98 115155 (2018).

[47] https://doi.org/10.5523/bris.1n5ra5bhdim102ct2wb6ehfojw.

[48] http://bris.ac.uk/acrc/.

[49] https://jp-minerals.org/vesta/en/. 